\title{
A Gallery of Simple Models from Climate Physics
}

\author{
Dirk Olbers
}

\begin{abstract}
The climate system of the earth is one of the most complex systems presently investigated by scientists. The physical compartments - atmosphere, hydrosphere and cryosphere - can be described by mathematical equations which result from fundamental physical laws. The other 'nonphysical' parts of the climate system, as e.g. the vegetation on land, the living beings in the sea and the abundance of chemical substances relevant to climate, are represented by mathematical evolution equations as well. Comprehensive climate models spanning this broad range of coupled compartments are so complex that they are mostly beyond a deep reaching mathematical treatment, in particular when asking for general analytical solutions. Solutions are obtained by numerical methods for specific boundary and initial conditions. Simpler models have helped to construct these comprehensive models, they are also valuable to train the physical intuition of the behavior of the system and guide the interpretation of the results of numerical models. Simple models may be stand-alone models of subsystems, such stand-alone general circulation models of the ocean or the atmosphere or coupled models, with reduced degrees of freedom and a reduced content of the physical processes. They exist in a wide range of structural complexity but even the simplest model may still be mathematically highly complicated due to nonlinearities of the evolution equations. This article presents a selection of such models from ocean and atmosphere physics. The emphasis is placed on a brief explanation of the physical ingredients and a condensed outline of the mathematical form.
\end{abstract}

\section{Contents}

1. Introduction 2

2. Fluid dynamics and thermodynamics 5

2.1. Equations of motion for ocean and atmosphere $\quad 7$

2.2. Coupling of ocean and atmosphere 11

2.3. Building a climate model 13

3. Reduced physics equations 13

3.1. The wave branches 14

To appear: Progress in Probability Vol. 49, Eds.: P. Imkeller and J. von Storch, Birkhäuser Verlag, p. 3-63. 
3.2. The quasigeostrophic branch 21

3.3. The geostrophic branch 22

3.4. Layer and reduced gravity models 24

4. Integrated models 26

4.1. Energy balance models and the Daisy World 26

4.2. A radiative-convective model of the atmosphere 28

4.3. The ocean mixed layer 30

4.4. ENSO models 32

4.5. The wind- and buoyancy-driven horizontal ocean circulation 35

4.6. The thermohaline-driven meridional ocean circulation 40

4.7. Symmetric circulation models of the atmosphere 42

5. Low-order models 43

5.1. Benard convection 44

5.2. A truncated model of the wind-driven ocean circulation 46

5.3. The low frequency atmospheric circulation $\quad 47$

5.4. Charney-DeVore models 50

5.5. Low order models of the thermohaline circulation 53

5.6. The delayed ENSO oscillator 56

ReferencesREFERENCES $\quad 57$

Coordinates and constants $\quad 62$

The forcing functions of the wave equations 62

\section{Introduction}

The evolution of the climate system is governed by physical laws, most of which arise from mechanical and thermodynamical conservation theorems applying to the fluidal envelopes of the earth. They constitute a coupled set of partial differential equations, boundary and initial conditions, of the form

$$
\frac{\partial}{\partial t} \mathcal{B} \varphi+\mathcal{L} \varphi+\mathcal{N}[\varphi, \varphi]=\Sigma
$$

The state of the system is described by $\varphi(\boldsymbol{X}, t)$ which generally is a vector function. There are linear or nonlinear differential operators $\mathcal{B}, \mathcal{L}, \mathcal{N}$ acting on the spatial dependence of $\varphi$, and $\Sigma$ denotes sources of the property $\varphi$. Externally prescribed forces and coefficients may also enter the operators $\mathcal{B}, \mathcal{L}$ and $\mathcal{N}$. Nonlinearity, indicated above by the $\mathcal{N}$-term, is an inherent and important property of climate dynamics. It mostly arises from transport of $\varphi$ by the fluid motion (e.g. advection of heat in the ocean and atmosphere by the fluid circulation which in turn depends on the distribution of temperature). Nonlinearity not only defeats solutions of complex models by analytical means. It also introduces a coupling in the broad range of scales of the climate system, and may lead to multiple equilibria and 
chaotic behavior, thus rendering a separation of the system into a manageable aggregate of subsystems difficult.

The conservation equations, coming from basic physics, govern motions for a vast range of space-time scales, and climate models of ocean and atmosphere circulation must necessarily disregard a high frequency-wavenumber part of the spectrum of motions to describe the evolution of a slow manifold. Climate physicists do this by averaging and filtering techniques. If (1) is considered the result of such procedures - i.e. if $\varphi$ represents an averaged and slowly varying state - the source term $\Sigma$ contains contributions from the field $\varphi^{\prime}$ representing the subrange of scales, generally referred to as turbulence, and terms which couple the resolved component $\varphi$ to the filtered variables $\chi$ (the fast manifold). The source would then be of the form

$$
\Sigma=-\overline{\mathcal{N}\left[\varphi^{\prime}, \varphi^{\prime}\right]}-\mathcal{N}_{1}[\chi, \varphi]-\mathcal{N}_{2}[\chi, \chi]+F,
$$

where the overbar indicates averaging over the turbulent components and $F$ is an external source. For practical reasons climate models must be closed with respect to the turbulence which is usually done by invoking some parameterization relating the mean turbulent source to the resolved fields,

$$
\overline{\mathcal{N}\left[\varphi^{\prime}, \varphi^{\prime}\right]}=\mathcal{P}[\gamma, \varphi] .
$$

The parameterization operator $\mathcal{P}$ may be nonlocal in space and time but in most practical cases one deals with simple local and linear relations with constant parameters $\gamma$. As an example, the divergence of turbulent fluxes of heat is frequently represented by Fickian diffusion.

In the view of a climate physicist, stochastic elements enter the problem (1), (2) and (3) where variables or coefficients appear which are not well known and should be considered as members of some random ensemble. Depending on the problem the random variable could represent the initial conditions $\varphi(\boldsymbol{X}, t=0)$, the external forcing $F$, the turbulent field $\varphi^{\prime}$ (in form of the parameters $\gamma$ ) or the fast manifold $\chi$ of the system.

Evidently, with this concept in mind, problem (1) is a nonlinear Langevin equation which was the starting point of Hasselmann's stochastic climate model (Hasselmann 1976). It was practically applied by Hasselmann and coworkers to explain the observed redness of climate spectra in terms of white noise forcing. Examples are the sea surface temperature variability on time scales of weeks to decades, treated by a Langevin model of ocean mixed-layer physics (Frankignoul and Hasselmann 1977), long-term climate variations of the global temperature, treated by a Langevin global energy balance model (Lemke 1977), and similar treatment of sea-ice variations (Lemke et al. 1980). A review of various applications has recently been given by Frankignoul (1995) and an even more recent 
investigation of that framework in the wind-driven ocean circulation is found in Frankignoul et al. (1997) and Frankignoul (1999).

Almost any compartment of the global climate system can be viewed through a stochastic frame. For each separate compartment one can identify an external driving force, and when this is varying in a stochastic way the system's response will be a stochastic process as well, with physically and mathematically interesting properties if the system has a rich interesting 'life', for instance in form of nonlinearities, resonances, time delay, instabilities and other ingredients of complex dynamics.

In the sections 2 and 3 I give a brief review of the basic fluid mechanical and thermodynamical equations used in ocean and atmosphere physics. There are various important aspects where geophysical fluid dynamics differ from conventional fluid mechanics. All models presented in the paper can be derived from this fundament, at least in principle. In section 4 I introduce the concept of filtering which breaks the equations into those describing fast and slow manifolds of evolution. Basically, the equations split into two subsets, representing fast modes of motion with adjustment mechanisms due to gravity, and slow modes whose time scales are governed by the differential rotation of the earth, as contained in the latitudinal dependence of the Coriolis frequency.

Every part of the climate system shows a rich variability in space and time, so besides filtering in time oceanographers and meteorologists have developed various techniques to reduce the spatial degrees of freedom. Most of these techniques are brute force actions: the system dynamics is integrated or averaged in some spatial directions - examples of integrated models are collected in section 5 - and/or the state vector of the system is expanded into a set of spatial structure functions with subsequent heavy truncation down to a manageable number of variables examples of these low-order models are collected in section 6 . Though being brute the techniques are applied in an intelligent way in order to include the interesting physical mechanisms and arrive at a meaningful physical system.

To my knowledge only very few of the dynamical systems collected in this 'gallery' have been investigated within a stochastic framework, for some of them the interest was originally addressed exclusively to steady state solutions. It should be clear that the models are extremely simple crooks used by climate physicists to move on the complex terrain of the climate system in the search for understanding of bits and pieces. Most of them, however, are still so complex that general analytical solutions are not known. In fact, they are stripped-down and simplified parts of complex climate models which are cast into numerical coding and solved on computers. Occasionally, numerical climate modelers have driven their codes by artificial white noise forcing to study the long-term red response (see e.g. Mikolajewicz and Maier-Reimer 1990, Eckert and Latif 1997).

Only models of ocean and atmosphere systems will be introduced. I will briefly explain their physics and point out where random elements might be attached. In most cases, however, this is obviously the prescribed forcing which in total or in part can be considered as a random variable. I should like to point 
out that the choice of the models is rather subjective and the presentation rather limited: the emphasis lies on the model equations rather than on physical or mathematical results. In any case, I strongly recommend to consult the original or text book literature for a deeper understanding of the model context and applicability. Suggestions for further reading are given in each section.

\section{Fluid dynamics and thermodynamics}

The evolution of the atmosphere or ocean is governed by the conservation of momentum, total and partial masses, and internal energy. The state of the system is completely described by a 7-dimensional state vector which is usually taken as $(\boldsymbol{V}, T, p, \varrho, m=[S$ or $q])$ where $\boldsymbol{V}$ is the 3-dimensional velocity of the fluid, $T$ the temperature, $p$ the pressure, $\varrho$ is the density of total mass, and $m$ the concentration of partial mass (such that $\varrho m$ is the density of the respective substance). For each of the fluids - seawater or air - there are only two dynamically relevant partial masses. In the ocean we have a mixture of pure water and various salts which are combined into one salinity variable $m=S$ (measured in $\mathrm{kg}$ salt per $\mathrm{kg}$ sea water). The air of the earth's atmosphere is considered as a mixture of dry air (basically oxygen and nitrogen) and water vapor with concentration $m=q$ (measured in $\mathrm{kg}$ vapor per $\mathrm{kg}$ moist air $)^{1}$. The concentration of the complementary partial mass is then $1-m$. For a binary fluid, i.e. a fluid composed from two partial masses, the thermodynamic state is described by three thermodynamic state variables which are usually taken as $T, m$ and $p$. This implies that any thermodynamic potential can be expressed in these three variables. In particular, since the density $\varrho$ is a thermodynamic variable, there is a relation

$$
\varrho \quad=F(T, m, p),
$$

which is referred to as equation of (thermodynamic) state. For the atmosphere, (4) is the ideal gas law, expressed in this context for the mixture of the two ideal gases dry air and water vapor. For the ocean, various approximate formulae are used (see e.g. Gill 1982).

The above mentioned conservation theorems may be expressed as a set of partial differential equations for the state vector, written here in a rotating coordinate system fixed to the earth, with angular velocity $\boldsymbol{\Omega}$, and with consideration of the gravitational and centrifugal acceleration combined in $\boldsymbol{g}$,

\footnotetext{
${ }^{1}$ Other constituents of the air such as water droplets, ice and radioactive trace gases can be neglected in the mass balance.
} 


$$
\begin{aligned}
\varrho \frac{D \boldsymbol{V}}{D t} & =-2 \varrho \boldsymbol{\Omega} \times \boldsymbol{V}-\nabla p+\varrho \boldsymbol{g}+\boldsymbol{F} \\
\frac{D \varrho}{D t} & =-\varrho \boldsymbol{\nabla} \cdot \boldsymbol{V} \\
\varrho \frac{D m}{D t} & =G_{m} \\
\varrho c_{p} \frac{T}{\theta} \frac{D \theta}{D t} & =\varrho c_{p}\left[\frac{D T}{D t}-\Gamma \frac{D p}{D t}\right]=G_{\theta} .
\end{aligned}
$$

The advection operator is

$$
\varrho \frac{D \varphi}{D t}=\varrho\left[\frac{\partial}{\partial t}+\boldsymbol{V} \cdot \nabla\right] \varphi=\frac{\partial}{\partial t}(\varrho \varphi)+\nabla \cdot(\boldsymbol{V} \varrho \varphi)
$$

where the first of these relations defines the time rate of change following the motion of a fluid parcel (Lagrangian or material derivative) and the second gives the equivalent Eulerian form where the effect of the flow appears now as the divergence of the advective flux $\boldsymbol{V} \varrho \varphi$ of the property $\varphi$. Equations (4) to (8) form a complete set of evolution equations.

In (8) the use of the potential temperature $\theta$ enables to express the conservation of internal energy in a simpler form by separating the adiabatic heating (derived from adiabatic expansion work contained in the second term in the brackets) and the diabatic heating rate $G_{\theta}$ in the heating rate of the ordinary (measured in-situ) temperature $T$. The potential temperature is defined by $d \theta=d T-\Gamma d p$ for an infinitesimal adiabatic displacement in the thermodynamic phase space. Here $\Gamma=\alpha T /\left(\varrho c_{p}\right)$ is the adiabatic temperature gradient, i.e. $d T / d p=\Gamma$ if $G_{\theta}=0$. The thermal expansion coefficient $\alpha$ and the specific heat $c_{p}$ are known thermodynamic functions of $T, m, p$. The potential temperature also depends on a constant reference pressure $p_{0}$; if the fluid parcel is moved adiabatically from its pressure $p$ to this reference pressure $p_{0}$ and temperature is measured there, its value equals the potential temperature $\theta$ of the parcel. The differential relation $d \theta=d T-\Gamma d p$ may be integrated to express the potential temperature in terms of the thermodynamic state variables in the form $\theta=\theta(T, m, p)$. This relation may be used to replace the ordinary temperature $T$ by $\theta$ to simplify the equations. Then, an equation of state $\varrho=G(\theta, m, p)=F(T(\theta, m, p), m, p)$ is appropriate. In fact when meteorologists or oceanographers refer to temperature in a dynamical model they usually mean potential temperature ${ }^{2}$.

${ }^{2}$ It should noted that the difference between $T$ and $\theta$ is small in oceanic conditions (about $0.5 \mathrm{~K}$ at most), it is usually ignored in the term on the lhs of (8). Furthermore, the specific heat $c_{p}$ in this term is taken at the value of the reference pressure while $c_{p}$ in the next part of the equation is taken at the in-situ pressure (for an ideal gas $c_{p}$ is constant so that this difference is irrelevant for the atmosphere). 
The source/sink terms $\boldsymbol{F}, G_{m}$ and $G_{\theta}$ of momentum, partial mass and internal energy contain an important part of the physics. In general we may write the source/sink terms as the sum of a flux divergence - which describes the transport of property through the boundaries of fluid parcels - and source/sink terms which are proportional to the volume, e.g. $G_{q}=-\boldsymbol{\nabla} \cdot \boldsymbol{J}_{q}+C_{q}$ where $\boldsymbol{J}_{q}$ is the diffusive flux of vapor $q$ and $C_{q}$ represents the source/sink due to evaporation of water droplets in clouds or condensation of vapor to liquid water. Boundary conditions will be discussed later in section 2.1 for a simplified set of equations.

When the fluxes appearing in $\boldsymbol{F}, G_{m}$ and $G_{\theta}$ are taken according to molecular theory Navier-Stokes equations are obtained as balance of momentum and Fickian diffusion of substances and heat is considered. In this case the equations (4) to (8) describe the full spectrum of atmospheric and oceanic motions, including sound waves with time scales of milliseconds to the thermohaline circulation of the ocean with periods of up to thousands of years. Clearly, such a range of variability is not the aim of a climate model: solving equations over climate time scales with resolution down to the sound waves is intractable and certainly not meaningful. Fortunately, the coupling of large-scale oceanic and atmospheric motions with motions at very small spatial and temporal scales of sound is very weak and can safely be ignored. The elimination of sound waves from the evolution equations and other approximations are outlined in the next section. Further wave filtering of (4) to (8) is demonstrated in the sections 3.1 and 3.2 .

\subsection{Equations of motion for ocean and atmosphere}

Climate is defined as an average of the state of ocean and atmosphere and the other parts of the climate system over space and time. A climate model of any complexity level will always have to abandon to resolve a certain range of small scales. It must cut off the resolved part of variability somewhere at the high frequencies and wavenumbers and we must look for a slow manifold of solutions. In geophysical fluid dynamics two concepts are employed to handle the cut-off procedure: filtering and averaging. Filtering eliminates some part of variability by analytical treatment of the equations of motion with the aim to derive equations which describe a slow manifold of solutions. In contrast, averaging is a brute force action: defining cut-off scales for space and time any field $\varphi(\boldsymbol{X}, t)$ is split into a mean $\bar{\varphi}(\boldsymbol{X}, t)$ over the subscale range, and the deviation $\varphi^{\prime}(\boldsymbol{X}, t)$ (the turbulent component). Then, equations are derived for the mean fields by averaging the original equations (named Reynolds averaging after O. Reynolds). Equations for higher order moments of $\varphi^{\prime}$ are considered as well to close the system. To simplify the work arising from the non-commutativity of averaging and differential operators, the averaging procedure is frequently formulated in terms of an ensemble of states $\varphi(\boldsymbol{X}, t ; \lambda)$ such that $\bar{\varphi}(\boldsymbol{X}, t)=\int d P(\lambda) \varphi(\boldsymbol{X}, t ; \lambda)$ is the expectation with respect to a probability measure $d P(\lambda)$ and $\varphi^{\prime}=\varphi-\bar{\varphi}$ is the deviation of a particular realization.

As a consequence of nonlinearity the averaged equations are not closed: the advection terms introduce divergences of fluxes $\overline{\boldsymbol{V}^{\prime} \varphi^{\prime}}$ supported by the motion in 
the subrange of scales. These Reynolds fluxes override the molecular fluxes by far (except in thin layers on the fluid boundary) and the latter are usually neglected. For ocean and atmosphere circulation models various elaborate closure schemes have been worked out to relate the Reynolds fluxes to resolved fields. Here, we shall only consider the simplest one: all Reynolds fluxes will be expressed by a diffusive parameterization,

$$
\overline{V^{\prime} \varphi^{\prime}}=-\mathbb{D} \cdot \nabla \bar{\varphi}
$$

with a diagonal diffusion tensor $\mathbb{D}=\operatorname{diag}\left(D_{h}, D_{h}, D_{v}\right)$. We omit the overbar of the mean fields in the following.

An obvious way to eliminate sound waves from the system is to consider the fluid as incompressible, i.e. to ignore the pressure dependence in the equation of state (4). Geophysical fluid dynamicists have less stringent approximations, the anelastic and the Boussinesq approximation. The density and pressure fields are expressed as a perturbation $\rho, \pi$ about a hydrostatically balanced $\operatorname{state}^{3} \varrho_{r}(z), p_{r}(z)$ such that $\varrho=\varrho_{r}(z)+\rho, p=p_{r}(z)+\pi$ and $d p_{r} / d z=-g \varrho_{r}$ and $p_{r}(z=0)=0$. For wave and QG problems (see sections 3.1 and 3.2 below) $\varrho_{r}(z)$ is the horizontal mean of density in the area of interest, or some standard profile. It is associated with some $\theta_{r}(z)$ and $m_{r}(z)$ such that $\varrho_{r}(z)=G\left(\theta_{r}(z), m_{r}(z), p_{r}(z)\right)$. In models which should predicted the complete stratification $\theta_{r}$ and $m_{r}$ (but not $\varrho_{r}$ ) are taken constant. The perturbation fields are generally small compared to the reference state variables.

Apparently, the pressure $p_{r}(z)$ is - together with the corresponding gravity force $g \varrho_{r}(z)$ - inactive in the momentum equations, they may there be eliminated. Sound waves are filtered by realizing that the time rate of change of density $\rho$ due to diabatic effects and compressibility is much smaller than that due to change of volume (given by the flow divergence). In the anelastic approximation the mass conservation (6) is then replaced by

$$
\boldsymbol{\nabla} \cdot\left(\varrho_{r} \boldsymbol{V}\right)=\varrho_{r} \boldsymbol{\nabla} \cdot \boldsymbol{V}+w \frac{d \varrho_{r}}{d z}=0
$$

where $w$ is the vertical component of the velocity vector $\boldsymbol{V}$. Notice that the equation of state - equation (4) is now expressed by the perturbation density $\rho$ - still describes the complete compressibility of the medium. Furthermore, the density $\varrho$

\footnotetext{
${ }^{3}$ The coordinate system is chosen with $z$-direction parallel and opposite to the gravity acceleration vector $\boldsymbol{g}$ and $z=0$ at the mean sea level. We will use 'horizontal' coordinates $\lambda$ and $\phi$ in a spherical coordinate system attached to the earth; $\lambda$ is longitude, $\phi$ is latitude. In the $\beta$-plane approximation used below these spherical coordinates are then approximated as local Cartesian coordinates by $d x=a d \phi, d y=a \cos \phi_{0} d \lambda$ where $a$ is the earth radius and $\phi_{0}$ the reference latitude.
} 
as factor in the inertial terms (all terms on the lhs of (5) to (8)) is replaced by the reference density $\varrho_{r}$.

There is a suite of further approximations which finally casts the equations (4) to (8) into the form representing the large-scale oceanic and atmospheric flow in climate models. These include:

- the hydrostatic approximation which realizes that pressure and gravity forces approximately balance in the vertical (not only for the reference fields but for the perturbation fields as well),

- the traditional approximation which drops all forces arising from the meridional component $\Omega \cos \phi$ of the angular velocity $\Omega=(0, \Omega \cos \phi, \Omega \sin \phi)$ of the earth.

Hiding the Reynolds fluxes and other sources in $\boldsymbol{\Phi}, \Gamma_{m}$ and $\Gamma_{\theta}$, the equation of motion for ocean and atmosphere then become in the anelastic approximation

$$
\begin{aligned}
\frac{D \boldsymbol{u}}{D t} & =-f \boldsymbol{k} \times \boldsymbol{u}-\frac{1}{\varrho_{r}} \nabla \pi+\boldsymbol{\Phi} \\
\frac{\partial \pi}{\partial z} & =-g \rho \\
\nabla \cdot\left(\varrho_{r} \boldsymbol{u}\right)+\frac{\partial}{\partial z}\left(\varrho_{r} w\right) & =0 \\
\varrho_{r} \frac{D m}{D t} & =\Gamma_{m} \\
\varrho_{r} c_{p} \frac{T}{\theta} \frac{D \theta}{D t} & =\Gamma_{\theta} \\
\rho & =G\left(\theta, m, p_{r}+\pi\right)-G\left(\theta_{r}, m_{r}, p_{r}\right) .
\end{aligned}
$$

Here $\boldsymbol{u}$ is the horizontal and $w$ the vertical velocity, $\boldsymbol{k}$ is a vertical unit vector, $f=2 \Omega \sin \phi$ is the Coriolis frequency, and $\nabla$ is the horizontal gradient or divergence operator. These equations are referred to as shallow water equations; when expressed in spherical coordinates of the earth and the radius is taken constant in the metric coefficients they are called primitive equations. Apart for the complete elimination of sound waves the shallow water system also deforms the kinematics of high frequency gravity waves.

The set (12) to (17) is one of many ways to represent the evolution equations of the atmosphere. Frequently, the complete mass conservation (6) is used instead of (14) - in particular in numerical models where the analytical convenience of simple equations is not needed - which takes into account an incomplete filtering of sound waves (the hydrostatic approximation alone filters only vertically propagating sound waves).

The last term in (11) is of order $g H / c_{r}^{2}$ where $c_{r}$ is the speed of sound of the reference profile and $H$ the depth of the fluid. The Boussinesq approximation is applied to the ocean where we find $g H / c_{r}^{2} \ll 1$. It omits therefore the $\varrho_{r}$-term in 
(11), arriving at $\boldsymbol{\nabla} \cdot \boldsymbol{V}=0$, and replaces $\varrho$ in the inertial terms by a constant $\varrho_{0}$ since density varies only little in the ocean. In addition the perturbation pressure is omitted from the equation of state (17). The Boussinesq equations then become

$$
\begin{aligned}
\frac{D \boldsymbol{u}}{D t} & =-f \boldsymbol{k} \times \boldsymbol{u}-\nabla \frac{\pi}{\varrho_{0}}+\mathbf{\Phi} \\
\frac{\partial}{\partial z}\left(\pi / \varrho_{0}\right) & =-g \rho / \varrho_{0} \\
\nabla \cdot \boldsymbol{u}+\frac{\partial w}{\partial z} & =0 \\
\varrho_{0} \frac{D m}{D t} & =\Gamma_{m} \\
\varrho_{0} c_{p} \frac{T}{\theta} \frac{D \theta}{D t} & =\Gamma_{\theta} \\
\rho & =G\left(\theta, m, p_{r}\right)-G\left(\theta_{r}, m_{r}, p_{r}\right) .
\end{aligned}
$$

The most important contributions to $\boldsymbol{\Phi}, \Gamma_{m}$ and $\Gamma_{\theta}$ for oceanic or atmospheric

flows arise from Reynolds fluxes of momentum, partial mass and heat in both media - generally expressed by diffusive laws - and additionally from the radiative flux and phase transitions of water in the atmosphere, thus

$$
\begin{aligned}
\boldsymbol{\Phi} & =\nabla \cdot\left(A_{h} \nabla \boldsymbol{u}\right)+\frac{\partial}{\partial z} A_{v} \frac{\partial \boldsymbol{u}}{\partial z} \\
\Gamma_{m} & =\nabla \cdot\left(K_{h} \nabla m\right)+\frac{\partial}{\partial z} K_{v} \frac{\partial m}{\partial z}+C_{m} /\left(\varrho_{r} c_{p}\right) \\
\Gamma_{\theta} & =\varrho_{r} c_{p}\left[\nabla \cdot\left(K_{h} \nabla \theta\right)+\frac{\partial}{\partial z} K_{v} \frac{\partial \theta}{\partial z}\right]-\nabla \cdot \boldsymbol{J}_{r a d}+\Lambda_{m} C_{m} .
\end{aligned}
$$

In the ocean the divergence of the radiative flux vector $\boldsymbol{J}_{\text {rad }}$ may be neglected below the top few meters. The terms involving $C_{m}$ appear only in the atmospheric balances, they describe the effect of evaporation $e$ and condensation $c$ of water with $C_{q}=e-c$. Furthermore, $\Lambda_{q}$ is the latent heat of evaporation. There is no source of salt in the ocean so that $C_{S} \equiv 0$. In the simplest form the (eddy) diffusion coefficients $A_{h}, A_{v}, K_{h}$ and $K_{v}$ are taken constant.

Finally, we should realize that - due to the hydrostatic approximation - the equations (12) to (17) do not describe vertical convection which occurs in the ocean by increasing the buoyancy by cooling or evaporation and in the atmosphere by decreasing the buoyancy by heating the air. For the latter case meteorologists have developed complex convection parameterizations whereas the oceanic case is treated quite simple by taking very large vertical diffusion coefficients to mimic the increased vertical mixing resulting from unstable stratification. 


\subsection{Coupling of ocean and atmosphere}

The boundary conditions of each medium must express the physical requirement of continuity of the fluxes of momentum, partial masses and internal energy across (and normal to) the boundaries. For instance, the net vertical heat flux leaving the atmosphere at the air-sea interface must be taken up by the advective and diffusive fluxes of heat in the ocean. We will describe the simple physical ideas of parameterization of boundary fluxes (for details cf. Gill 1982, Peixoto and Oort 1992).

If the topography of the sea surface is ignored - for simplicity we make this approximation in this section - the continuity of the heat flux at the interface at $z=0$ of ocean and atmosphere would be expressed by $J_{\theta}\left(z=0^{+}\right)=J_{\theta}(z=$ $0^{-}$) where $J_{\theta}$ is the total vertical flux of internal energy. The simple diffusive parameterization of $J_{\theta}$ and the other fluxes in (24) are, however, not valid in the proximity of the air-sea interface as gradients of properties may become very small due to the action of enhanced turbulence. Meteorologists have developed alternative and more accurate parameterizations of surface fluxes in terms of 'bulk formulae'. Observations have shown that vertical fluxes of momentum, matter and energy are constant within a shallow layer of a few meters above the surface and empirical laws have been elaborated to relate these fluxes to the values of velocity, partial masses and temperature at the upper boundary of this 'constant flux layer' (the standard level is 10 meters height) and the corresponding sea surface properties.

The conductive heat flux $Q_{H}$ is parameterized by the difference of surface air and water temperature, and a similar relation is taken for the rate of evaporation $E$,

$$
Q_{H}=\varrho_{a i r} c_{p} C_{H} U_{a i r}\left(\theta_{s}-\theta_{a i r}\right) \quad E=\varrho_{a i r} C_{E} U_{a i r}\left(q_{s}-q_{a i r}\right)
$$

with dimensionless coefficients $C_{H}$ and $C_{E}$ of order $10^{-3}$. The variables $\theta_{\text {air }}, q_{\text {air }}$ and $U_{\text {air }}$ are the air temperature, specific humidity and wind speed, taken at the standard level, $E$ is the rate of evaporation/condensation (in $\mathrm{kg}$ water vapor per $\mathrm{m}^{2}$ and $s$ ), and $q_{s}$ the saturation value of humidity at temperature $\theta_{s}$. The momentum flux is parameterized by a drag law relating the tangential surface stress - the wind stress - to the $10 \mathrm{~m}$ wind speed in the form

$$
\boldsymbol{\tau}_{0}=\varrho_{a i r} C_{W} U_{a i r} \boldsymbol{u}_{a i r}
$$

again with a drag coefficient $C_{W} \sim 10^{-3}$.

The only driving of the climate system occurs by the radiative heat flux coming from the sun and entering the atmosphere at its outer edge with a value of $S_{0}=1372 \mathrm{Wm}^{-2}$, the solar 'constant' which is, however, not constant on long time scales because of changing orbital parameters of the earth, and on small time 
scales because of changing solar activity. The heat flux from the earth interior is negligible. At the interface of the atmosphere and the ocean, as well as atmosphereland, heat is exchanged by radiation, i.e. short-wave solar radiation and long-wave radiation. The latter is determined by the surface temperature and thus mainly in the infrared range. Furthermore, there is heat loss associated with evaporation (the 'latent' heat flux $Q_{L}=\Lambda_{q} E$ ) and by heat conduction (the 'sensible' heat flux $\left.Q_{H}\right)$. Above the constant flux layer and below it in the ocean the fluxes are carried further as parameterized by the diffusive approximations ${ }^{4}$. The sum of the above described heat fluxes has to match the oceanic diffusive heat flux at sea surface,

$$
-\left[\varrho c_{p} K_{v} \frac{\partial \theta}{\partial z}\right]_{\text {ocean }}=-Q_{S W}\left(1-\alpha_{s}\right)+Q_{L W}+Q_{L}+Q_{H}
$$

A similar relation holds for the atmospheric flux above the constant flux layer. Here, $Q_{S W}$ is the incident energy flux of short-wave radiation (computed from a radiation model which is an essential part of a full climate model, see section 4.2), $\alpha_{s}$ is the sea surface albedo, $Q_{L W}=\epsilon \sigma T_{s}^{4}$ follows from the Stefan-Boltzmann law ( $\epsilon$ is the emissivity of the surface, $\sigma$ the Stefan-Boltzmann constant and $T_{s}$ the sea surface temperature).

The vapor entering the atmosphere by evaporation is carried further by turbulence so that

$$
-\left[\varrho K_{v} \frac{\partial q}{\partial z}\right]_{a t m}=E .
$$

Exchange of other partial masses is irrelevant, in particular the total (diffusive plus advective) flux of salt through the sea surface is assumed to vanish,

$$
-\left[\varrho K_{v} \frac{\partial S}{\partial z}\right]_{\text {ocean }}=S(P-E)
$$

Here, $P$ is the rate of precipitation (in $\mathrm{kg}$ water per $\mathrm{m}^{2}$ and second and $(P-E)$ equals the total mass flux entering the ocean from the atmosphere. Notice that (29) expresses the vanishing of the sum of the advective and diffusive salt fluxes across the air-sea interface. In the ocean the stress exerted by the wind is transferred from the surface by turbulent diffusion, thus

$$
\left[\varrho A_{v} \frac{\partial \boldsymbol{u}}{\partial z}\right]_{\text {ocean }}=\varrho_{a i r} C_{W} U_{a i r} \boldsymbol{u}_{a i r}
$$

\footnotetext{
${ }^{4}$ For simplicity we take the diffusive parameterization for the oceanic fluxes in this section. A flavor of the complex physics of the oceanic side of the air-sea interface is discussed in section 4.3 .
} 
At the upper boundary of the atmosphere and the bottom of the ocean the usual conditions assume the vanishing of the normal fluxes of partial masses and heat. The requirement of zero flux of total mass implies that the normal velocity vanishes at the ocean bottom. There is a suite of differing stress conditions, with the limiting cases of no-slip (i.e. vanishing of the tangential velocity) and free-slip (i.e. vanishing of the tangential stress).

\subsection{Building a climate model}

A complete climate model needs more than ocean and atmosphere modules. As mentioned above, a radiation model is needed which calculates the short- and longwave radiation field in the atmosphere from the incoming solar flux at the top of the atmosphere. A sea-ice module is needed to simulated the freezing/melting and storage of frozen water as well as the transport of sea ice. Boundary conditions over land surfaces and sea ice are required, possibly including a hydrological model which organizes water storage on land and transport by rivers into the sea. For long-term climate simulations the building and decay of ice-sheets must be included. Various empirical parameters have to be specified, as e.g. the albedo of the land and sea ice surface, turbulent diffusivities and exchange coefficients. One even has demand for model components of the terrestrial and marine ecosystems, atmospheric chemistry and ocean biogeochemical tracers. A complete climate model should ultimately even include interactions between socioeconomic variables and climate.

Climate physicists have developed a wide suite of techniques to circumvent the enormous problems of treating the complete system. They

- use stand-alone models of the compartments of the climate system,

- simplify the dynamics,

- decrease the degrees of freedom

to arrive at manageable and understandable systems. In the following we find various examples of such undertaking.

Further reading for section 2: Gill (1982), Müller and Willebrand (1986), Washington and Parkinson (1986), Peixoto and Oort (1992), Trenberth (1992), Olbers et al. (1999)

\section{Reduced physics equations}

A dynamical system governed by an equation like (1) can be transformed to a state space in which the linear part of the evolution operator, i.e. $\mathcal{B}^{-1} \mathcal{L}$ for (1), is diagonal. For not too nonlinear fluid systems this implies the isolation of the internal time scales appearing in the system's evolution, which mean a classification of the wave branches. The following sections are largely dedicated to this problem. 


\subsection{The wave branches}

In wave theory of fluid motions the balance of partial mass and potential temperature are conveniently combined into a balance of buoyancy, determined from (15) to (17). Taking for simplicity the Boussinesq approximation the buoyancy is $b=-g \rho / \varrho_{0}$. Separating out nonlinear advection terms, the linearized equations of motion become

$$
\begin{aligned}
\frac{\partial u}{\partial t}-f v+\frac{\partial p}{\partial x} & =\mathcal{F}^{u} \\
\frac{\partial v}{\partial t}+f u+\frac{\partial p}{\partial y} & =\mathcal{F}^{v} \\
\frac{\partial b}{\partial t}+w N^{2} & =\mathcal{G}_{b} \\
\frac{\partial p}{\partial z}-b & =0 \\
\nabla \cdot \boldsymbol{u}+\frac{\partial w}{\partial z} & =0,
\end{aligned}
$$

where we have absorbed the constant density $\varrho_{0}$ in the pressure ${ }^{5}$. The terms on the rhs contain the nonlinearities and the source/sink terms ${ }^{6}$ in the corresponding equations (12) to (16), e.g. $\left(\mathcal{F}^{u}, \mathcal{F}^{v}\right)=\boldsymbol{\Phi} / \varrho_{0}-\boldsymbol{u} \cdot \nabla \boldsymbol{u}-w \partial \boldsymbol{u} / \partial z$. The Brunt-Väisälä frequency $N(z)$, defined by

$$
N^{2}=-\frac{g}{\varrho_{0}} \frac{d \varrho_{r}}{d z}-\frac{g^{2}}{c_{r}^{2}}=g\left[\alpha_{*} \frac{d \theta_{r}}{d z}-\beta_{*} \frac{d S_{r}}{d z}\right]
$$

is the only relic of the stratification of the reference density field ( $\alpha_{*}$ and $\beta_{*}$ are the coefficients of thermal and haline expansion when the equation of state is expressed in terms of potential temperature). Atmosphere and ocean are wave guides because of the vertical boundaries and the mean stratification entering the theory via $N(z)$. For purpose of demonstration we will consider here the ocean case with the kinematic boundary conditions $D \zeta / D t-w=E-P$ at the sea surface $z=\zeta$, and $w=-\boldsymbol{u} \cdot \nabla h$ at the bottom $z=-h$, and the dynamic boundary condition $p=p_{a t m}$ at $z=\zeta$. Here, the rate of evaporation minus precipitation, $E-P$, and the atmospheric pressure $p_{a t m}$ enter as external forcing. We expand these conditions about the mean sea surface $z=0$ and the mean bottom $z=-H$ and get

\footnotetext{
${ }^{5}$ We use the Boussinesq form of the scaled pressure $\pi / \varrho_{0} \rightarrow p$ and density $\rho / \varrho_{0} \rightarrow \rho$ in the rest of this paper. Also stresses and surface mass fluxes will be scaled by $\varrho_{0}$ so that stresses and pressure are measured in units of $\mathrm{m}^{2} \mathrm{~s}^{-2}$ and surface mass fluxes in $\mathrm{ms}^{-1}$

${ }^{6}$ All source/sink terms - written in calligraphic type - appearing on the rhs in the linearized equations in this section are given in the appendix.
} 


$$
\begin{aligned}
\frac{\partial \zeta}{\partial t}-w & =\mathcal{Z} & & \text { at } z=0 \\
p-g \zeta & =\mathcal{P} & & \text { at } z=0 \\
w & =\mathcal{W} & & \text { at } z=-H,
\end{aligned}
$$

where $\mathcal{Z}, \mathcal{P}, \mathcal{W}$ contain the forcing terms and the nonlinear terms arising in this expansion (see appendix). Notice that $H$ is constant.

The wave state is described by a 3 -d state vector. Taking $(u, v, p)$ as state vector the remaining fields follow from diagnostic equations: (34) determines $b$, (35) together with the kinematic bottom boundary condition determines $w$, and (38) determines $\zeta$ as functionals of $(u, v, p)$. A prognostic equation for the pressure to supplement (31) and (32) is obtained from (33), (34) and (37) to (39). After some mathematical work one arrives at

$$
\frac{\partial p}{\partial t}+M \nabla \cdot \boldsymbol{u}=\mathcal{Q},
$$

where the operator

$$
M=g \int_{-H}^{0} d z^{\prime \prime}+\int_{z}^{0} d z^{\prime} N^{2}\left(z^{\prime}\right) \int_{-H}^{z^{\prime}} d z^{\prime \prime}
$$

acts only on the vertical structure. The eigenvalue problem $M \varphi=c_{n}^{2} \varphi$ is of the standard Liouville form (a differential form with appropriate boundary conditions is easily derived). There is a discrete spectrum $\left(c_{n}^{2}, n=0,1,2,3 \cdots\right)$ with an orthogonal and complete set of eigenfunctions $\varphi_{n}(z)$. The eigenvalue $c_{n}^{2}$ determines the $n$-th Rossby radius $\lambda_{n}=c_{n} / f$. The eigenvalues should be ordered according to $c_{0}>c_{1}>\cdots$, then $\lambda_{0} \approx \sqrt{g H} / f$ (this is the barotropic mode $\varphi_{0}$ which is almost vertically constant) and $\lambda_{1} \approx \sqrt{g^{\prime} H} / f$ with the 'reduced gravity' $g^{\prime}=g \Delta \varrho_{r} / \varrho_{0}$ (this is the first baroclinic mode $\varphi_{1}$ which has one zero in the vertical, $\Delta \varrho_{r}$ is the vertical range of $\varrho_{r}$ ). It is found that the barotropic Rossby radius $\lambda_{0}$ exceeds the baroclinic radii $\lambda_{i}, i \geq 1$ by far since $g^{\prime} \ll g$.

3.1.1. Midlatitude Waves For midlatitude waves it turns out that the projection of the prognostic equations (31), (32) and (40) onto the wave branches requires to form the divergence $\delta$ and vorticity $\eta$ of the horizontal momentum balances, and therefore

$$
\delta=\frac{\partial u}{\partial x}+\frac{\partial v}{\partial y} \quad \text { and } \quad \eta=\frac{\partial v}{\partial x}-\frac{\partial u}{\partial y}
$$


are used to replace ${ }^{7} u=\nabla^{-2}(\partial \delta / \partial x-\partial \eta / \partial y), v=\nabla^{-2}(\partial \eta / \partial x+\partial \delta / \partial y)$. The evolution equations become

$$
\begin{aligned}
\frac{\partial \eta}{\partial t}+f \delta+\beta v & =\frac{\partial \mathcal{F}^{v}}{\partial x}-\frac{\partial \mathcal{F}^{u}}{\partial y} \\
\frac{\partial \delta}{\partial t}-f \eta+\beta u+\nabla^{2} p & =\frac{\partial \mathcal{F}^{u}}{\partial x}+\frac{\partial \mathcal{F}^{v}}{\partial y} \\
\frac{\partial p}{\partial t}+M \delta & =\mathcal{Q}
\end{aligned}
$$

where $\beta=d f / d y$ arises from the differential rotation due to the change of the Coriolis parameter $f$ with latitude ${ }^{8}$. Projection onto a scalar wave equation is easily done for the so called $f$-plane case with uniform rotation where $f=f_{0}$ is considered constant and $\beta=0$. Then, for the pressure,

$$
\frac{\partial}{\partial t}\left[\frac{\partial^{2}}{\partial t^{2}}+f_{0}^{2}\left(1-\mathcal{M} \nabla^{2}\right)\right] p=\frac{\partial^{2} \mathcal{Q}}{\partial t^{2}}+f_{0}^{2} \mathcal{M}\left[\frac{\partial \mathcal{D}}{\partial t}-f_{0} \mathcal{C}\right]
$$

with $\mathcal{M}=M / f_{0}^{2}$ is obtained. Plane wave solutions $p \sim \varphi(z) \exp i(\boldsymbol{K} \cdot \boldsymbol{x}-\omega t)$ with $\boldsymbol{K}=(k, \ell)$ are found for the unforced equation with three branches (i.e. different wave types). The dispersion relations are $\omega_{n}^{(1,2)}= \pm f_{0}\left[1+\left(K \lambda_{n}\right)^{2}\right]^{1 / 2}$ (the gravity branches) and $\omega_{n}^{(3)}=0$ (the geostrophic branch). The latter is degenerated on the $f$-plane: it describes a steady geostrophically balanced current where Coriolis and pressure forces balance, $-f_{0} v=-\partial p / \partial x$ and $f_{0} u=-\partial p / \partial y$. The degeneracy is relieved on the $\beta$-plane where both $f$ and $\beta$ are retained and considered constant in the above equations. This approximation can be justified by a proper expansion into various small parameters (see QG approximation in the next section); here we simply assume $\beta L / f \ll 1$ where $L$ is a typical horizontal scale, and $\omega \ll f$. The first order correction of the geostrophic branch can then be derived by extracting the geostrophic balances from (31) and (32), evaluating the geostrophic vorticity as $\eta=\left(1 / f_{0}\right) \nabla^{2} p$ and using (43) and (45) to get

$$
\frac{\partial}{\partial t}\left(1-\mathcal{M} \nabla^{2}\right) p-\beta \mathcal{M} \frac{\partial p}{\partial x}=f_{0} \mathcal{C}
$$

We deduce $\omega_{n}^{(3)}=-\beta k /\left[k^{2}+\ell^{2}+\lambda_{n}^{-2}\right]$ which is the dispersion relation of linear planetary Rossby waves. Another way to filter out gravity waves is to neglect the tendency in the divergence equation (44).

\footnotetext{
${ }^{7}$ The use of the inverse of the Laplace operator $\nabla^{2}$ in this section and other operators involving $\nabla^{2}$ is the sloppy notation of a physicist.

${ }^{8}$ We use here the $\beta$-plane approximation which projects the equations onto a plane tangent to the earth at a central latitude $\phi_{0}$ but has a nonuniform rotation: the Coriolis parameter is expanded as $f=f_{0}+\beta y$ with $f_{0}=2 \Omega \sin \phi_{0}, \beta=(2 \Omega / a) \cos \phi_{0}$ and $x, y$ are Cartesian coordinates.
} 
The separation of the wave spectrum of motions is not completed with the above analysis: (46) describes all wave branches though one is degenerate while (47) yields the correct form of the geostrophic wave response. A complete separation into the wave branches requires a proper diagonalization of the linear matrix operator appearing in the system (43) to (45). With the same approximations used above to get (47) this goal can strictly be achieved. Let us consider the wave evolution equations, written in terms of the state vector

$$
\psi_{1}=\delta \quad \psi_{2}=\eta \quad \psi_{3}=\frac{1}{f_{0}} \nabla^{2} p .
$$

It is found in the form ${ }^{9}$

$$
\frac{\partial \psi_{a}}{\partial t}+i\left(H_{a b}+B_{a b}\right) \psi_{b}=q_{a} \quad a=1,2,3
$$

where $q_{a}$ derives from the above source terms and operators

$$
H_{a b}=-i f_{0}\left(\begin{array}{ccc}
0 & -1 & 1 \\
1 & 0 & 0 \\
\mathcal{M} \nabla^{2} & 0 & 0
\end{array}\right) \quad B_{a b}=-i \beta \nabla^{-2}\left(\begin{array}{ccc}
\partial / \partial x & -\partial / \partial y & 0 \\
\partial / \partial y & \partial / \partial x & 0 \\
0 & 0 & 0
\end{array}\right)
$$

appear. Because $B_{a b} / H_{a b}=O\left(\beta L / f_{0}\right)$ (where $L$ is a typical length scale) and $H_{a b}$ is easily diagonalized we will approach the problem by expansion in terms of $\beta L / f_{0}$. To lowest order in $\beta L / f_{0}$ the eigenvalue problem of the evolution operator is $H_{a b} \boldsymbol{R}_{b}^{s}=\Omega^{(s)} \boldsymbol{R}_{a}^{s}$ (no $s$-summation) which is solved by

$$
\begin{array}{r}
\Omega^{(s)}=s \Omega=s f_{0}\left(1-\mathcal{M} \nabla^{2}\right)^{1 / 2} \\
s= \pm, 0 \\
\boldsymbol{R}_{1}^{s}=i s \Omega / f_{0} \quad \boldsymbol{R}_{2}^{s}=1 \quad \boldsymbol{R}_{3}^{s}=1-\left(s \Omega / f_{0}\right)^{2}
\end{array}
$$

The branches $s= \pm$ describe gravity waves and $s=0$ is the steady geostrophic flow. The $\boldsymbol{R}_{a}^{s}$ achieve the representation of the state vector $\psi_{a}$ in terms of the wave branch vector $\psi^{s}$, i.e. $\psi_{a}=\boldsymbol{R}_{a}^{s} \psi^{s}$. The inverse transformation is achieved by left eigenvectors of the evolution operator, $\boldsymbol{P}_{a}^{s} H_{a b}=\Omega^{(s)} \boldsymbol{P}_{b}^{s}$. which are found to be

$$
\begin{aligned}
{\left[\boldsymbol{P}_{a}^{ \pm}\right] } & =\frac{1}{2}\left(\Omega / f_{0}\right)^{-2}\left[-i \Omega / f_{0}, 1,-1\right]^{T} \\
{\left[\boldsymbol{P}_{a}^{0}\right] } & =\left(\Omega / f_{0}\right)^{-2}\left[0,\left(\Omega / f_{0}\right)^{2}-1,1\right]^{T} .
\end{aligned}
$$

\footnotetext{
${ }^{9}$ Summation over repeated lower or upper indices is used in the following.
} 
Projection onto the wave state is thus performed by $\psi^{s}=\boldsymbol{P}_{a}^{s} \psi_{a}$. The representation and projection operators are mutually orthogonal, $\boldsymbol{P}_{a}^{s} \boldsymbol{R}_{a}^{t}=\delta^{s t}, \boldsymbol{R}_{a}^{s} \boldsymbol{P}_{b}^{s}=\delta_{a b}$. Finally, the first order correction of the eigenvalue problem is $B_{a b} \boldsymbol{R}_{b}^{s}+H_{a b} \tilde{\boldsymbol{R}}_{b}^{s}=$ $\Omega^{(s)} \tilde{R}_{a}^{s}+\tilde{\Omega}^{(s)} \boldsymbol{R}_{a}^{s}$ where the $B_{a b}$ and the tilde terms are first order in $\beta L / f_{0}$. It yields corrections to the representation operator (which we do not use) and $\tilde{\Omega}^{(s)}=\boldsymbol{P}_{a}^{s} B_{a b} \boldsymbol{R}_{b}^{s}$ (no $s$-summation). For the geostrophic branch we thus get

$$
\tilde{\Omega}^{(0)}=\frac{i \beta \mathcal{M}}{1-\mathcal{M} \nabla^{2}} \frac{\partial}{\partial x}
$$

as correction to the geostrophic evolution operator. Denoting from now $\tilde{\Omega}^{(0)}$ by $\Omega^{(0)}$ the wave evolution is then governed by the three diagonal problems

$$
\frac{\partial \psi^{s}}{\partial t}+i \Omega^{(s)} \psi^{s}=q^{s} \quad s= \pm, 0 .
$$

These abstract equations of wave evolution can be cast into a more familiar form. It can easily be verified that $\psi=\nabla^{-2} \psi^{0}$ is the streamfunction of the geostrophic part of the motion and $f_{0} \psi$ is the corresponding pressure part. From (54) we find

$$
\frac{\partial}{\partial t}\left(\nabla^{2}-\mathcal{M}^{-1}\right) \psi+\beta \frac{\partial \psi}{\partial x}=\mathcal{C}
$$

for the $s=0$-equation, describing the evolution of the Rossby wave branch. The gravity wave branch fields are conjugate to each other, $\psi^{+}=\left(\psi^{-}\right)^{*}$. Introducing real and imaginary parts by $\psi^{+}+\psi^{-}=\varpi$ and $\psi^{+}-\psi^{-}=\gamma$ the evolution of the gravity branch is found as

$$
\begin{aligned}
\frac{\partial^{2} \varpi}{\partial t^{2}}+f_{0}^{2}\left(1-\mathcal{M} \nabla^{2}\right) \varpi & =\left(\frac{\Omega}{f_{0}}\right)^{-2} \frac{\partial \mathcal{C}}{\partial t}-f_{0} \mathcal{D} \\
\frac{\partial^{2} \gamma}{\partial t^{2}}+f_{0}^{2}\left(1-\mathcal{M} \nabla^{2}\right) \gamma & =-\left(\frac{\Omega}{f_{0}}\right)^{-1}\left\{\frac{\partial \mathcal{D}}{\partial t}+f_{0} \mathcal{C}\right\} .
\end{aligned}
$$

The gravity wave pressure is given by $f_{0} \mathcal{M} \varpi$ and the total pressure thus by $p=$ $f_{0}(\mathcal{M} \varpi+\psi)$.

For practical applications the branch amplitude fields $\psi$ and $\varpi, \gamma$ should be expanded in the eigenfunctions of $\mathcal{M}$, which simply replaces $\mathcal{M}$ by $\lambda_{n}^{2}$ and e.g. the Rossby wave field $\psi(x, y, z, t)$ by the modal amplitudes $\psi_{n}(x, y, t)$. Kinematic conditions of zero normal velocity, $\boldsymbol{n} \cdot \boldsymbol{u}=0$, must be satisfied at solid 
boundaries. Separate equations for long or short waves may be generated by assuming $\mathcal{M} \nabla^{2} \sim(K \lambda)^{2} \ll 1$ for length scales which are large compared to the respective Rossby radius, or $\mathcal{M} \nabla^{2} \sim(K \lambda)^{2} \gg 1$ for the opposing case.

3.1.2. Equatorial waves At the equator the Coriolis parameter vanishes and a special wave theory must be developed. The equatorial $\beta$-plane uses $f=\beta y$ with constant $\beta=2 \Omega / a$ in (31), (32) and (40). The system supports gravity and Rossby type waves which are trapped vertically as in midlatitudes but also meridionally. Equations for the wave branches are obtained by vertical decomposition (replacement of $M$ by $c_{n}^{2}$; we will omit the index $n$ ). Scaling of time by $(2 \beta c)^{1 / 2}=\sqrt{2} c / \lambda_{e}$ and space coordinates by $(c / 2 \beta)^{1 / 2}=\lambda_{e} / \sqrt{2}$ and use of the state vector $(q, v, r)$, with $q=p / c+u$ and $r=p / c-u$, is convenient. Here $\lambda_{e}=(c / \beta)^{1 / 2}$ is the equatorial Rossby radius (of vertical mode $n$ ). Then

$$
\begin{array}{r}
\left(\frac{\partial}{\partial t}+\frac{\partial}{\partial x}\right) q+\mathcal{E}^{+} v=\mathcal{F}^{u}+\mathcal{Q}=\mathcal{F} \\
\frac{\partial v}{\partial t}+\frac{1}{2}\left(\mathcal{E}^{-} q+\mathcal{E}^{+} r\right)=2 \mathcal{F}^{v}=\mathcal{H} \\
\left(\frac{\partial}{\partial t}-\frac{\partial}{\partial x}\right) r+\mathcal{E}^{-} v=-\mathcal{F}^{u}+\mathcal{Q}=\mathcal{G}
\end{array}
$$

with

$$
\mathcal{E}^{ \pm}=\frac{\partial}{\partial y} \mp \frac{1}{2} y .
$$

Notice the following properties:

$$
\begin{array}{r}
\mathcal{E}=\mathcal{E}^{+} \mathcal{E}^{-}-\frac{1}{2}=\mathcal{E}^{-} \mathcal{E}^{+}+\frac{1}{2}=\frac{\partial^{2}}{\partial y^{2}}-\frac{1}{4} y^{2} \\
\mathcal{E} D_{\ell}=-\left(\ell+\frac{1}{2}\right) D_{\ell} \quad D_{\ell}(y)=2^{-\ell / 2} e^{-\frac{1}{4} y^{2}} H_{\ell}(y / \sqrt{2}) \\
\quad \ell=0,1,2, \cdots \\
\mathcal{E}^{+} D_{\ell}=-D_{\ell+1} \quad \mathcal{E}^{-} D_{\ell}=\ell D_{\ell-1} .
\end{array}
$$

The $D_{\ell}(y)$ and $H_{\ell}(y)$ are parabolic cylinder functions and Hermite polynomials, respectively. The operators $\mathcal{E}^{ \pm}$thus excite or annihilate one quantum of the meridional mode index $\ell$. Apparently, to satisfy (57) to (58), we must take $v \sim D_{\ell}, r \sim$ $D_{\ell-1}, q \sim D_{\ell+1}$. Wave solution $(q, v, r) \sim\left(\boldsymbol{q}, \boldsymbol{v} \mathcal{E}^{-}, \boldsymbol{r}\left(\mathcal{E}^{-}\right)^{2}\right) D_{\ell}(y) \exp i(k x-\omega t)$ are obtained which are oscillatory in a band of width of the Rossby radius about the equator but decay exponentially away from there. These are $\omega_{0}=k$ (Kelvin wave), $\omega_{1}=k / 2+\left(k^{2} / 4+1 / 2\right)^{1 / 2}$ (Yanai wave) and for $\ell \geq 2$ we have $\omega^{2}-k^{2}-(1 / 2) k / \omega=$ $\ell-1 / 2$ with approximate solutions $\omega= \pm\left(k^{2}+\ell+1 / 2\right)^{1 / 2}$ (two gravity waves) and 
$\omega_{\ell}=-k /\left(2 k^{2}+2 \ell-1\right)$ (Rossby wave). The corresponding eigenvectors $(\boldsymbol{q}, \boldsymbol{v}, \boldsymbol{r})$ are easily evaluated. Notice that Kelvin and Yanai waves travel eastward and Rossby waves westward while gravity waves exist for both directions.

There is a simple procedure to filter out the gravity and Yanai waves from the system: this is performed by omitting the time derivative in (58). Eliminating $v$ from (57) and (59) yields a prognostic equation

$$
\begin{array}{r}
\mathcal{E} \frac{\partial \varphi}{\partial t}-\frac{1}{2} \frac{\partial \varphi}{\partial x}= \\
=\left(\mathcal{E}+\frac{1}{2}\right) \mathcal{E}^{-} \mathcal{F}-\left(\mathcal{E}-\frac{1}{2}\right) \mathcal{E}^{+} \mathcal{G}-\left(\frac{1}{2} \frac{\partial}{\partial t}+\mathcal{E} \frac{\partial}{\partial x}\right) \mathcal{H}
\end{array}
$$

for the equatorial potential vorticity variable $\varphi=\mathcal{E}^{-} q-\mathcal{E}^{+} r=y p+2 \partial u / \partial y$. Because $\varphi \equiv 0$ for the Kelvin wave, only long Rossby waves (with $\omega=-k /(2 \ell-1)$ ) are retained in (62). The Kelvin wave has $v=r \equiv 0$ and is therefore described by a simple equation for the amplitude $q_{0}(x, t)$,

$$
\left(\frac{\partial}{\partial t}+\frac{\partial}{\partial x}\right) q_{0}=\mathcal{F}_{0}
$$

By projecting onto the meridional modes various problems of trapped equatorial wave motion can be formulated. If the system is zonally unbounded or periodic the waves propagate independently. Interesting problems arise in a zonally bounded wave guide (e.g. the Pacific Ocean) since wave reflection at zonal boundaries couple the wave branches. The reflection process is complicated. Depending on the frequency it may involve a large number of modes of long and short waves (at the eastern boundary the solution even requires further coastal trapped waves). We abandon an exact treatment to give a simple example: assume that the only waves present are Kelvin waves (traveling eastward with speed $\omega / k=1$ ) and the long Rossby waves (traveling westward with speed $\omega / k=-1 / 3$ ) of mode number $\ell=2$ and consider an ocean contained zonally in the interval $x=0$ and $x=x_{e}$. The Rossby wave amplitude $\varphi_{2}(x, t)$ and the Kelvin wave amplitude $q_{0}(x, t)$ are coupled by requiring zero flux of mass through the boundaries. Because the zonal velocity is given by $u=(q-r) / 2$ we get the constraint (assuming $H=0$ in (58))

$$
\left(\frac{1}{3} d_{3}+d_{1}\right) \varphi_{2}+d_{0} q_{0}=0 \quad \text { at } x=0, x_{e}
$$

where $d_{\ell}=\sqrt{2^{\ell} \pi \ell !}$ is the normalization constant of $D_{\ell}$. The system is then governed by (62), projected on $\ell=2$, and (63), they are coupled by (64). These waves dominate the ocean component of ENSO (see the sections 4.4 and 5.6). 
Further reading: Hasselmann (1976), Gill (1982), Olbers (1986), Frankignoul et al. (1997)

\subsection{The quasigeostrophic branch}

The evolution equation (55) of Rossby waves is the linearized version of the quasigeostrophic $(\mathrm{QG})$ potential vorticity equation ${ }^{10}$

$$
\frac{d Q}{d t}=\frac{\partial Q}{\partial t}+\boldsymbol{u} \cdot \nabla Q=\operatorname{curl} \boldsymbol{\Phi}+f_{0} \frac{\partial}{\partial z} \frac{\mathcal{G}_{b}}{N^{2}}
$$

which states the conservation of the QG potential vorticity ${ }^{11}$

$$
Q=\nabla^{2} \Psi+\frac{\partial}{\partial z} \frac{f_{0}^{2}}{N^{2}} \frac{\partial \Psi}{\partial z}+f_{0}+\beta y
$$

along the path of the fluid elements (the path is projected onto the horizontal plane because vertical advection can be neglected for QG motions). The advection velocity is given by the geostrophic part of the current, $\boldsymbol{u}=\boldsymbol{k} \times \nabla \Psi$ where $\Psi=$ $p / f_{0}$ is the QG streamfunction. The constituents of the QG potential vorticity (66) are identified with the relative vorticity of the horizontal velocity, the stretching vorticity and the planetary vorticity. Eq. (65) may be derived from (55) by noting that $Q=-\mathcal{M}^{-1}\left(1-\mathcal{M} \nabla^{2}\right) \psi^{0}+f_{0}+\beta y$. Indeed, extracting the geostrophic advection term and the frictional and buoyancy sources from the source term on the rhs of (55) (and neglecting the remaining terms) we get (65). A more precise derivation starts with (31) to (35) in spherical coordinates and performs an expansion in various small parameters. The most important are the Rossby number $\operatorname{Ro}=U /(2 \Omega L)$, the planetary scale ratio $L / a$, and the aspect ratio $H / L$ and Ekman numbers Ek $=A_{h} /\left(2 \Omega L^{2}\right)$ or $A_{v} /\left(2 \Omega H^{2}\right)$, where $U, H, L$ are scales of the horizontal velocity and vertical and horizontal lengths, $\Omega$ is the angular velocity of the earth, and $a$ is the radius of the earth. For QG theory all these parameters are assumed to be of the same small magnitude. The theory is thus restricted to a geostrophic horizontal flow (to zero order) with a small vertical circulation (the vertical velocity $w=-\left(f_{0} / N^{2}\right) d(\partial \Psi / \partial z) / d t$ is first order) and a slowly evolving geostrophic pressure field governed by (65) which is a first order balance. Boundary conditions at top and bottom follow from (37) to (39), they are expressed by

\footnotetext{
${ }^{10}$ curl, acting on a 2-d vector, is a sloppy notation for curl $\mathbf{\Phi}=\partial \Phi^{(y)} / \partial x-\partial \Phi^{(x)} / \partial y$.

${ }^{11} \mathrm{QG}$ theory is presented here for a Boussinesq fluid. For the anelastic approximation the stretching term in the potential vorticity has an extra factor $1 / \varrho_{r}(z)$ in front of the first vertical derivative and a factor $\varrho_{r}(z)$ behind.
} 


$$
\begin{aligned}
\frac{d}{d t} \frac{\partial \Psi}{\partial z}+\frac{N^{2}}{g} \frac{d \Psi}{d t}=\frac{\mathcal{G}_{b}}{N^{2}}-\frac{N^{2}}{g f_{0}} \frac{d p_{a t m}}{d t} \quad \text { at } z=0 \\
\frac{d}{d t} \frac{\partial \Psi}{\partial z}=\frac{N^{2}}{f_{0}} \boldsymbol{u} \cdot \nabla h \quad \text { at } z=-h .
\end{aligned}
$$

The condition at the upper boundary is expanded about the mean sea surface $z=0$, the condition at the bottom is exact (though $\nabla h$ should be small of order of the Rossby number).

QG motions are a projection of the complete flow onto a slow manifold. The equations could be supplemented by the so far neglected sources arising from the fast manifold, basically the gravity wave field. It should also be emphasized that more elaborate theories exist for slow manifolds. They arise from a different ordering of the small parameters mentioned above.

Further reading: Pedlosky (1986)

\subsection{The geostrophic branch}

The most important nonlinearity in the dynamics of large scale flow stems from the advection of heat and partial mass. The balance of momentum is well described by the geostrophic and hydrostatic equations as utilized in the quasigeostrophic theory. While this theory aims to describe perturbations on a given background, a theory of the establishment of the oceanic stratification must consider the complete balance of advection and diffusion for temperature and partial mass. In the atmosphere convection and heating by phase transitions and by radiation is of overwhelming importance. Theories of the thermohaline stratification of the ocean in general simplify the problem by using the perturbation density $\rho$ as thermohaline variable and ignore its compressibility. Expressed in spherical coordinates we are dealing then with the planetary geostrophic equations (in Boussinesq form)

$$
\begin{aligned}
-f v & =-\frac{1}{a \cos \phi} p_{\lambda} \\
f u & =-\frac{1}{a} p_{\phi} \\
p_{z} & =-g \rho \\
\frac{1}{\cos \phi}\left[u_{\lambda}+(v \cos \phi)_{\phi}\right]+w_{z} & =0 \\
\rho_{t}+\frac{u}{a \cos \phi} \rho_{\lambda}+\frac{v}{a} \rho_{\phi}+w \rho_{z} & =\left(K_{v} \rho_{z}\right)_{z}+\nabla \cdot\left(K_{h} \nabla \rho\right),
\end{aligned}
$$


where we use indices to denote partial derivatives. As noted by Needler (1967) these equations may be reduced to a single nonlinear differential equation for the pressure. A simpler form was found by Welander (1971), defining the $M$-function

$$
M(\lambda, \phi, z)=\int_{z_{0}}^{z} p d z^{\prime}+M_{0}(\lambda, \phi),
$$

which allows to express all fields as partial derivatives of one variable,

$$
\begin{array}{rlrl}
p & =M_{z} & \rho & =-\frac{1}{g} M_{z z} \\
u & =-\frac{1}{f a} M_{\phi z} & v & =\frac{1}{f a \cos \phi} M_{\lambda z} .
\end{array}
$$

The vertical velocity may as well be expressed by $M$ : from (71) we find the planetary vorticity relation $f w_{z}=\beta v$ and thus, by integration,

$$
w=\frac{\beta}{f} \int_{0}^{z} v d z^{\prime}+w_{0}=\frac{\beta}{f^{2} a \cos \phi}\left(M-M_{0}\right)_{\lambda}+w_{0},
$$

where $w_{0}(\lambda, \phi)=w\left(\lambda, \phi, z_{0}\right)$. Thus, with $M_{0}=2 \Omega a^{2} \sin ^{2} \phi \int_{0}^{\lambda} w_{0} d \lambda^{\prime}$, the thermohaline density equation (72) results in a nonlinear partial differential equation of second degree and fourth order for $M$,

$$
\begin{array}{r}
a^{2} \Omega \sin 2 \phi M_{z z t}+\frac{\partial\left(M_{z}, M_{z z}\right)}{\partial(\lambda, \phi)}+\cot \phi M_{\lambda} M_{z z z}= \\
=a^{2} \Omega \sin 2 \phi\left[\left(K_{v} M_{z z z}\right)_{z}+\nabla \cdot\left(K_{h} \nabla M_{z z}\right)\right] .
\end{array}
$$

The $M$-equation is thought to describe the evolution of the oceanic thermocline as response to pumping of water with surface characteristics $\rho_{s}(\lambda, \phi)=\rho\left(\lambda, \phi, z_{0}\right)$ to depth, at a rate given by the pumping velocity $w_{0}$. The level $z_{0}$ is placed at the bottom of the turbulent layer which is immediately influenced by wind and surface wave breaking (roughly the upper 50 to 100 meters, see section 4.3). The pumping velocity is then determined by the divergence of the wind-induced transport in that layer, i.e. $w_{0}$ is the Ekman pumping velocity

$$
w_{0}=w_{E}=\operatorname{curl} \frac{\boldsymbol{\tau}_{0}}{f},
$$

where $\boldsymbol{\tau}_{0}$ is the wind stress. Boundary conditions for (75) are thus 


$$
\begin{array}{r}
M_{\lambda z}=2 \Omega a^{2} \sin ^{2} \varphi w_{E}(\lambda, \varphi) \quad \text { and } \quad M_{z z}=-g \rho_{s}(\lambda, \varphi) \quad \text { at } z=z_{0} \\
M_{\lambda}=0 \quad \text { and } \quad M_{z z z}=0 \quad \text { at } z=-H
\end{array}
$$

assuming for simplicity a flat bottom. The last two conditions express the vanishing of $w$ and the buoyancy flux at the bottom.

Further reading: Pedlosky (1987), Salmon (1998)

\subsection{Layer and reduced gravity models}

Special treatment of the vertical dependence of field variables was demonstrated in section 3.1 where we have used decomposition into vertical normal modes. Another popular projection of the overwhelmingly horizontally layered structure of ocean and atmosphere is that of layer models. In the simplest concept the fluid is considered as a stack of immiscible layers, each with a constant density ${ }^{12} \varrho_{i}$. The index $i=1$ (=top layer $), \cdots, n$ (=bottom layer) identifies the layer of vertical height $d_{i}(\boldsymbol{x}, t)$ with vertically constant horizontal velocity $\boldsymbol{u}_{i}=\boldsymbol{u}_{i}(\boldsymbol{x}, t)$. This can be justified by the Taylor-Proudman theorem according to which vertical shears $\partial \boldsymbol{u}_{i} / \partial z$ are weak if the fluid is homogeneous, rapidly rotating and hydrostatic. The pressure $p_{i}$ is evaluated from the hydrostatic balance (13) as sum of the masses in the layers on top of the respective one so that $\boldsymbol{\nabla} p_{i}=g \sum_{j<i} \varrho_{j} \nabla d_{j}$. The evolution of the system is then governed by the conservation of momentum and mass, in the flux form this is expressed by

$$
\begin{array}{r}
\frac{\partial}{\partial t} d_{i} \boldsymbol{u}_{i}+\nabla \cdot\left(\boldsymbol{u}_{i} d_{i} \boldsymbol{u}_{i}\right)+f \boldsymbol{k} \times d_{i} \boldsymbol{u}_{i}=-d_{i} \boldsymbol{\nabla} p_{i}+\boldsymbol{\tau}_{i-1}-\boldsymbol{\tau}_{i}+\boldsymbol{R}_{i} \\
\frac{\partial}{\partial t} d_{i}+\nabla \cdot d_{i} \boldsymbol{u}_{i}=0,
\end{array}
$$

where $\boldsymbol{\tau}_{i}$ is the horizontal stress at the bottom of the $i$-th layer, $\boldsymbol{\tau}_{0}$ is the stress at the surface of the fluid (the wind stress in case of the ocean), and $\boldsymbol{R}_{i}$ denotes the divergence of lateral stresses, $\boldsymbol{R}_{i}=A_{h} \nabla^{2} d_{i} \boldsymbol{u}_{i}$ for simple lateral eddy diffusion of momentum, $\boldsymbol{R}_{i}=-\epsilon_{i-1} d_{i-1}\left(\boldsymbol{u}_{i}-\boldsymbol{u}_{i-1}\right)-\epsilon_{i} d_{i}\left(\boldsymbol{u}_{i}-\boldsymbol{u}_{i+1}\right)$ for linear interfacial friction where $\epsilon_{0}=0$ and $\boldsymbol{u}_{n+1}=0$ for the bottom layer $i=n$. The coefficient

\footnotetext{
${ }^{12}$ The concept of immiscible layers should actually be based on an adiabatically conserved property such as potential temperature or potential density. The latter is defined as $\varrho_{\theta}=G\left(\theta, m, p_{0}\right)$, i.e. the density taken for the parcel's potential temperature and partial mass but at the reference pressure $p_{0}$, using the equation of state outlined in section 2. Both properties, $\theta$ and $\varrho_{\theta}$, are conserved along streamlines if diabatic processes - such as diffusion, radiative heating etc - are absent. In-situ density is not conserved but enters the calculation of the pressure force. This conflict between in-situ and potential density is inherent for all layer models.
} 
$\epsilon_{n}$ describes bottom friction. The simplest stratified model has two layers with $d_{1}=\zeta+\xi, d_{2}=h-\xi$ where $\zeta$ is the elevation of the surface and $z=-\xi$ and $z=-h$ give the positions of the layer interface and the bottom, respectively.

The ocean abyss has a very sluggish flow. Applied to the ocean circulation, the assumption of a motionless deep layer is therefore a coarse but fairly acceptable approximation in certain areas. It yields the reduced gravity model where $\boldsymbol{u}_{2} \equiv$ $0, \boldsymbol{\tau}_{2} \equiv 0$ requires $\nabla p_{2} \equiv 0$ or $g \zeta=g^{\prime} \xi$ with $g^{\prime}=g\left(\varrho_{2}-\varrho_{1}\right) / \varrho_{1}$ (reduced gravity). The resulting model

$$
\begin{array}{r}
\frac{\partial}{\partial t} \boldsymbol{u}_{1}+\boldsymbol{u}_{1} \cdot \boldsymbol{\nabla} \boldsymbol{u}_{1}+f \boldsymbol{k} \times \boldsymbol{u}_{1}=-g^{\prime} \boldsymbol{\nabla} \xi+\boldsymbol{\tau}_{0} / d_{1}+\boldsymbol{R}_{1} \\
\frac{\partial}{\partial t} \xi+\nabla \cdot \xi \boldsymbol{u}_{1}=0
\end{array}
$$

is equivalent to the first vertical mode of the wave model (31), (32) and (40) if these equations are supplemented by the nonlinear terms.

For frictionless conditions the conservation of the potential vorticity $\left(\eta_{i}+\right.$ $f) / d_{i}$ with relative vorticity $\eta_{i}=\partial v_{i} / \partial x-\partial u_{i} / \partial y$ is easily proven from (78). Straightforward QG perturbation theory yields the layered version of the QG potential vorticity theory. For a two-layer system we find

$$
\begin{array}{r}
\frac{\partial Q_{i}}{\partial t}+\boldsymbol{u}_{i} \cdot \nabla Q_{i}=F_{i} / H_{i} \quad i=1,2 \\
Q_{1}=\nabla^{2} \Psi_{1}-\frac{f_{0}}{H_{1}}[\zeta-\xi]+f_{0}+\beta y \\
Q_{2}=\nabla^{2} \Psi_{2}-\frac{f_{0}}{H_{2}}[\xi-b]+f_{0}+\beta y
\end{array}
$$

where $\zeta=\left(f_{0} / g\right) \Psi_{1}, \xi=\left(f_{0} / g^{\prime}\right)\left(\Psi_{2}-\Psi_{1}\right)$ are the elevations of the surface and the interface, respectively, and $\Psi_{i}$ is the streamfunction $\left(\boldsymbol{u}_{i}=\boldsymbol{k} \times \boldsymbol{\nabla} \Psi_{i}\right)$. Furthermore, $H_{1}$ is the undisturbed thickness of the upper layer and $H_{2}-b$ the thickness of the lower layer with $b(\boldsymbol{x})$ as topographic elevation. The forcing and friction are contained in the source terms $F_{i}$. For forcing a QG ocean by wind stress one uses $F_{1}=$ curl $\boldsymbol{\tau}_{0}$, linear bottom friction has the form $F_{2}=-\epsilon_{2} H_{2} \nabla^{2} \Psi_{2}$. Additional interfacial friction is needed in case that the model does not adequately resolve the quasigeostrophic turbulence. Then a term $-\epsilon_{1} H_{1} \nabla^{2}\left(\Psi_{1}-\Psi_{2}\right)$ must appear in $F_{1}$ and a corresponding term with opposite sign in $F_{2}$. The QG model is also applied to the zonal atmospheric circulation. The domain is then zonally periodic and the forcing specified by relaxation of the interface to a prescribed zonal flow in form of meridional temperature or interface displacement $\xi_{R}(y)$. In this case, $F_{i}=\mp f_{0}\left[\xi-\xi_{R}(y)\right] / t_{R}$.

Further reading: Pedlosky (1986), Wolff et al. (1991), Pavan and Held (1996) 


\section{Integrated models}

Integrating any of the conservation equations, discussed in section 2 , over any piece of volume of the system yields a budget of the corresponding physical quantity $\varphi$ in that volume in terms of storage (rate of change of the content of $\varphi$ in the volume), fluxes across the boundaries and sources in the interior. It is also meaningful to consider budgets which apply to integration over certain spatial directions, say the vertical direction or the horizontal domain. In general, one cannot expect that such a reduction of the spatial degrees of freedom would yield a problem which is mathematically well-posed for determination of the integrated state variables, i.e. parameterizations must be considered to get a problem which is closed with respect to integrated variables. The method of spatial integration (or averaging) is nonetheless a frequently used crook to produce simplified models. The methodological similarity and overlapping with the low-order models discussed further below is obvious.

\subsection{Energy balance models and the Daisy World}

The most popular and simplest climate model reflects the energy balance of the earth in integrated form. The zero-dimensional model integrates the balance of heat (essentially (16) with boundary conditions discussed in section 2.2) vertically and laterally over the whole earth, such that the incoming and reflected solar radiation must balance the outgoing infrared radiation,

$$
c^{*} \frac{\partial T}{\partial t}=\frac{1}{4} S_{0}\left(1-\alpha_{p l}\right)-\epsilon^{*} \sigma T^{4} .
$$

Here, $T$ is thought to represent the mean surface temperature and the heat capacity $c^{*}=\varrho c_{p} H$ is adjusted to some global mean value, $H$ is a measure of the vertical extent of the climate system, $S_{0}$ is the solar constant (downward solar flux at the outer rim of the atmosphere), and $\alpha_{p l}$ the albedo. In the radiative cooling the Stefan-Boltzmann law is corrected by the factor $\epsilon^{*}<1$ to account for the difference between $T$ and the radiative temperature of the earth, it can also be used to correct for the greenhouse effect (then $\epsilon^{*} \approx 0.62$ ). The model is easily extended to a onedimensional version by omitting the meridional integration, the energy balance (81) then includes a term to represent the divergence of the meridional transport of heat. These models have extensively been reviewed in the literature (e.g. North 1981, Ghil 1981, see also chapters by Fraedrich and Imkeller in this book).

The interesting physics and mathematical complexity enter the models via feedback and nonlinearity implemented into the albedo dependence $\alpha_{p l}(T)$ on tem-

perature (in the one-dimensional case the albedo depends on the latitude of the ice extent and this depends on the local temperature). But the albedo would not only change if the surface becomes ice-covered, it also changes with changing plant cover. A very popular and philosophically stimulating model has been formulated 
by Lovelock to illustrate the Gaia Hypothesis (cf. Watson and Lovelock 1983) according to which the biosystem on earth creates its own - eventually optimal climate.

Consider a planet covered partly with white daisies (fraction $F_{w}$ ), black daisies (fraction $F_{b}$ ) and bare land (fraction $F_{\ell}$ ) such that $F_{\ell}+F_{w}+F_{b}=1$ and the albedo becomes $\alpha_{p l}=\alpha_{\ell} F_{\ell}+\alpha_{w} F_{w}+\alpha_{b} F_{b}$. The areas change in time due to growth and death of daisies. To put it more specific, we take Lotka-Volterra dynamics to describe these processes,

$$
\begin{aligned}
\frac{\partial F_{w}}{\partial t} & =-\gamma F_{w}+\beta\left(T_{w}\right) F_{\ell} F_{w} \\
\frac{\partial F_{b}}{\partial t} & =-\gamma F_{b}+\beta\left(T_{b}\right) F_{\ell} F_{b} .
\end{aligned}
$$

Here, $\gamma$ is the death rate (it can be taken constant) and $\beta$ the growth rate which depend on the local temperature, e.g. a function with optimal growth at $T_{o p t}$ is

$$
\beta(T)=1-\left(\frac{T-T_{o p t}}{T_{*}}\right)^{2}
$$

but other more complicated forms could be taken. The time in (82) is scaled by the value of the optimal growth rate. The energy balance is given by (81) but we include additionally a lateral heat exchange $N_{i}$ of each compartment $i=w, b, \ell$ with the environment, thus $N_{w} F_{w}+N_{b} F_{b}+N_{\ell} F_{\ell}=0$. The $N_{i}$ must be related to other variables in the problem. Lovelock considers the parameterization $N_{i}=q^{*}\left(\alpha_{i}-\alpha_{p l}\right)$ which yields

$$
\begin{aligned}
c \frac{\partial T_{i}}{\partial t} & =S\left(1-\alpha_{i}\right)-N_{i}-\epsilon^{*} \sigma T_{i}^{4}= \\
& =S\left(1-\alpha_{p l}\right)+q\left(\alpha_{p l}-\alpha_{i}\right)-\epsilon^{*} \sigma T_{i}^{4} \quad i=w, b, \ell
\end{aligned}
$$

where $c$ takes into account the time scaling of (82), and $S=S_{0} / 4$ and $q=$ $q^{*}+S$. The value of $q$ controls the importance of the heat exchange: for $q=0$ one gets a steady state where the heat exchange produces a uniform temperature, for $q=S$ one gets a local equilibrium with zero exchange, i.e. $N_{i}=0$. Thus $0 \leq q \leq S$ is the interesting range and the stationary points of (82) and (84) are easily determined. The thermostat property of the daisies can be demonstrated by numerical integration. Typical parameter values of this model are $\gamma=0.3, T_{o p t}=$ $295.5 \mathrm{~K}, T_{*}=17.5 \mathrm{~K}, q=0.2 S$.

The Daisy World could be used to bring more life into the higher dimensional energy balance models. Other interesting dynamics are obtained by coupling to 
an ice sheet module which, however requires one or two spatial dimensions (see e.g. Källen et al. 1980).

Further reading: Lemke (1977), Lovelock (1989), Olbers et al. (1997)

\subsection{A radiative-convective model of the atmosphere}

The balance of the horizontally averaged internal energy of the atmosphere is obtained from (16) and (24). For simplicity we take an atmosphere consisting of dry air, thus ignoring phase transitions. Written in terms of the horizontally averaged potential temperature $\theta(z, t)$ we get

$$
\varrho c_{p} \frac{\partial \theta}{\partial t}=-\frac{\partial J_{r a d}}{\partial z}+\frac{\partial}{\partial z} \varrho c_{p} K_{v} \frac{\partial \theta}{\partial z},
$$

Here, $J_{r a d}$ is the vertical flux of radiant energy. In the radiative-convective models the diffusive term should lead to a very efficient mixing if the air column is convectively unstable and thus, it is generally assumed that $K_{v}=0$ where $\partial \theta / \partial z>0$ (stable stratification) and $K_{v} \neq 0$ with a very large value where $\partial \theta / \partial z<0$ (unstable stratification).

The flux $J_{\text {rad }}$ follows from a radiative transfer equation determining the radiant intensity $I_{\nu}(\varphi, \vartheta)$ at each frequency $\nu$. The amount of radiant energy traversing a unit area per unit time from the solid angle $d \omega=\sin \vartheta d \vartheta d \varphi$ in the frequency interval $(\nu, \nu+d \nu)$ is given by $I_{\nu} d \nu d \omega$. To obtain the flux $J_{\text {rad }}$ the intensity has to be integrated over frequencies and solid angle, after projection of the rays onto the vertical direction,

$$
J_{r a d}=\int_{0}^{\infty} d \nu \int_{0}^{2 \pi} d \varphi \int_{0}^{\pi} d \vartheta \sin \vartheta \cos \vartheta I_{\nu}
$$

We assume for simplicity that scattering can be neglected. The radiative transfer equation then reduces to the Schwarzschild equation

$$
\cos \vartheta \frac{\partial}{\partial z} I_{\nu}=\varrho \kappa_{\nu}\left[-I_{\nu}+B_{\nu}(T)\right]
$$

where $\kappa_{\nu}$ is the absorption coefficient of the air (it is a sum over the cross sections of all radiatively active gases weighted by their relative mass fractions in the atmosphere) and $B_{\nu}(T)$ is Planck's function of the intensity of black body radiation at temperature $T$. Equation (87) describes the attenuation of the intensity along the vertical direction due to absorption, and the gain of radiant energy due to radiation from the heated air.

The temperature profile is then obtained by simultaneous solution of (85) and (87), using Poisson's formula $\theta=T\left(p_{0} / p\right)^{\gamma}$ to relate the in-situ temperature with the potential temperature $\left(\gamma=R / c_{p}=2 / 7\right.$ where $R$ is the gas constant and 
$p_{0}$ a constant reference pressure). The problem is highly complicated because the absorption coefficient is a very complicated function of frequency, reflecting a vast number of narrow absorption bands of the atmospheric gas constituents.

We consider a highly simplified radiation model, the grey atmosphere where $\kappa_{\nu}=\kappa$ is assumed constant. Integrating (87) over the frequencies of the long-wave (infrared) radiant energy, the horizontal plane and then separately over the upper part $0<\vartheta<\pi / 2$ and the lower part $\pi / 2<\vartheta<\pi$ the two-stream model is obtained

$$
\begin{aligned}
& \frac{\partial F^{\uparrow}}{\partial z}=\beta \varrho \kappa\left[-F^{\uparrow}+\sigma T^{4}\right] \\
& \frac{\partial F^{\downarrow}}{\partial z}=\beta \varrho \kappa\left[F^{\downarrow}-\sigma T^{4}\right] .
\end{aligned}
$$

The factor $\beta=5 / 3$ arises due to an approximation of the integrals (the problem is not closed exactly), and $F^{\uparrow}$ and $F^{\downarrow}$ are the total upward and downward flux of long-wave radiant energy, respectively, such that $J_{\text {rad }}=F^{\uparrow}-F^{\downarrow}+F_{S W}$, where the last summand is the net contribution from the solar (short-wave) radiation. Boundary conditions for the two-stream model are easily given: at the top of the atmosphere $(z=\infty)$ the downward flux is zero and at the bottom $(z=0)$ the upward flux is given by the infrared radiation of the surface, thus

$$
\begin{array}{ll}
F^{\downarrow}=0 & \text { at } z=\infty \\
F^{\uparrow}=\sigma T_{s}^{4} & \text { at } z=0,
\end{array}
$$

where $T_{s}=T(z=0)$ is the surface temperature. The boundary conditions for the heat balance (85) are simply $K_{v} \partial \theta / \partial z=0$ at the top and (27), expressed here for the atmospheric side,

$$
-K_{v} \varrho c_{p} \frac{\partial \theta}{\partial z}=F^{\uparrow}-F^{\downarrow}+F_{S W}=\sigma T_{s}^{4}-F^{\downarrow}+F_{S W} \quad \text { at } z=0 .
$$

Finally, a model of the solar part must be specified. The simplest version assumes that $F_{S W}$ penetrates undiminished from top to bottom and thus $F_{S W}=$ $-\left(1-\alpha_{p l}\right) S_{0} / 4$ where $\alpha_{p l}$ is the planetary albedo. A more complex but still simple model assumes an exponential decrease of the short-wave radiation, $\partial F_{S W} / \partial z=$ $-\kappa_{S W} F_{S W}$, with upper boundary condition $F_{S W}(z=\infty)=-\left(1-\alpha_{p l}\right) S_{0} / 4$. It is easy to show that a temperature profile in equilibrium with the radiation must 
yield zero net radiant energy flux at the top, thus $F^{\uparrow}(z=\infty)=\left(1-\alpha_{p l}\right) S_{0} / 4$. Notice that the solar constant $S_{0}$ is the only driving force of this model.

Further reading: Ramanathan and Coakley (1978), Salby (1996)

\subsection{The ocean mixed layer}

The limitation of applicability of the parameterizations (24) in the proximity of the sea surface has been touched in section 2.2 where, for the atmospheric side, the concept of the constant flux layer was introduced. Also oceanographers have developed much more elaborate parameterizations of the fluxes in the oceanic layer adjacent to the sea surface. Diffusive parameterizations do not work there because the layer is very well mixed by the action of the wind and breaking surface waves. Nevertheless, heat and substances pass this layer to enter the ocean interior.

The structure of the oceanic near-surface layer is well represented by a completely mixed fluid of temperature $T_{0}$ of vertical extent from the surface $z=0$ to a depth $z=-h$, residing over stratified water which, immediately below the mixed layer, has a thin sharp thermocline (a layer with strong vertical gradient of temperature), followed by a more gradual decrease of temperature down to the abyss. The structure is thus given by $T=T_{0}(t)$ for $0 \leq z \leq-h(t)$, and a linear increase of $T$ from $T_{0}$ to $T_{\star}$ between $z=-h(t)$ and $z=-h_{\star}$ to represent the thermocline. The deep temperature $T_{\star}$ must be specified in this model, the value $\Delta=h_{\star}-h$ is assumed constant and small, $\Delta \ll h$. It will not enter the model equations explicitly. The physics of the mixed layer model should determine $T_{0}(t)$ and $h(t)$ from the specified surface heat flux and the characteristics of the turbulence in that layer.

We step back to the Reynolds form of turbulent transports and concentrate on the heat balance (a more extended version may consider the salt budget in addition)

$$
\frac{\partial T}{\partial t}=\frac{\partial Q}{\partial z}
$$

where $Q=-\overline{w^{\prime} T^{\prime}}$ is the turbulent flux of heat (apart from a factor $\varrho c_{p}$ ). Evidently, since $T$ is constant in the mixed layer, $Q(z)$ must be a linear function of depth,

$$
Q(z)=Q_{0}+\frac{z}{h}\left(Q_{0}-Q_{h}\right)
$$

where $Q_{0}$ is the surface heat flux and $Q_{h}=Q(z=-h)$. The surface heat flux is a prescribed forcing, the flux $Q_{h}$ at the mixed layer base will be parameterized in a rather indirect way as shown below. Integrating (91) over the mixed layer we find

$$
h \frac{\partial T_{0}}{\partial t}=Q_{0}-Q_{h}
$$


whereas the integration over the intermediate layer $-h<z<-h_{\star}$ yields approximately

$$
\left(T_{0}-T_{\star}\right) \frac{\partial h}{\partial t}=Q_{h}-Q_{\star}
$$

We assume here that $Q_{\star}=Q\left(z=-h_{\star}\right)$ is small, it could easily be retained as a diffusive flux below the mixed layer. In practice, $T_{0}-T_{\star}$ will always be positive. Thus, according to (94), the mixed layer will deepen if the heat flux $Q_{h}$ is positive, i.e. downward. In this case, fluid of temperature $T_{\star}$ from below the mixed layer is warmed to $T_{0}$ and mixed ('entrained') into the mixed layer. As we shall see below, this mixing consumes turbulent energy, or - to be more specific - it can only take place if turbulence energy is available for mixing fluid from below into the mixed layer.

Now, the basic assumption of mixed layer physics is that $Q_{h}$ originates from turbulent processes above and should therefore not become negative: there is no cooling of fluid in the mixed layer from below and no 'unmixing'. Instead, if $Q_{h}$ drops to zero, there is not enough turbulent energy to mix at all at the mixed layer base. Mixing and deepening of the mixed layer then stops and a new mixed layer depth is established at a shallower depth. Its depth is determined from $Q_{h}=$ $f c t[h, \cdots]=0$ where $f c t[h, \cdots]$ is the parameterization of the flux $Q_{h}$ in terms of $h$ and other parameters which determine the mixing properties. This function is found from the budget of turbulent kinetic energy $T K E=u^{\prime 2} / 2$ in the mixed layer, given by

$$
\frac{\partial}{\partial t} T K E=\frac{\partial F}{\partial z}-\alpha g Q-\epsilon
$$

where $F=-\overline{\left(u^{\prime 2} / 2+p^{\prime}\right) w^{\prime}}$ is the turbulent flux of $T K E, \epsilon$ is the dissipation of $T K E$, and $\alpha g Q=-\alpha g \overline{w^{\prime} T^{\prime}}=-\overline{w^{\prime} b^{\prime}}$ is the exchange of TKE with the turbulent potential energy ( $\alpha$ is the coefficient of thermal expansion). Remember from section 3.1 that $b^{\prime}=-g \rho^{\prime}=\alpha g T^{\prime}$ is the buoyancy fluctuation (salinity is here neglected). Lifting of fluid with density anomaly $\rho^{\prime}>0$ increases the potential energy at the expense of $T K E$, then $\overline{w^{\prime} \rho^{\prime}}>0$ and thus $\alpha g Q>0$, as incorporated in (95). The $T K E$ usually equilibrates within a few minutes and integration of (95) over the mixed layer then yields, using (92),

$$
0=F_{0}-F_{h}-\alpha g \frac{h}{2}\left(Q_{0}+Q_{h}\right)-\int_{-h}^{0} \epsilon d z
$$

A few further assumptions (parameterizations) close the problem: $F_{h}$ is assumed small and neglected, $F_{0}$ is related to the wind stress $\tau_{0}$ (which excites surface waves which by breaking create $T K E$ ). By dimensional arguments one postulates $F_{0}=$ 
$c\left|\tau_{0}\right|^{3 / 2}$ with a dimensionless coefficient $c$ of order unity. Finally, the dissipation term must be positive and it is simply assumed that it 'eats' away a certain fraction of $F_{0}$ (which is positive) and the term involving the surface heat flux $Q_{0}$. This is a gain of $T K E$ for cooling $\left(Q_{0}<0\right)$ because the potential energy of the cooled and heavier fluid is converted to $T K E$. There is no eating from the $Q_{0}$-term in case of heating. Hence, the dissipation term is expressed as

$$
\int_{-h}^{0} \epsilon d z=r_{1} F_{0}+r_{2} \alpha g \frac{h}{4}\left(\left|Q_{0}\right|-Q_{0}\right)
$$

with $0<r_{1}, r_{2}<1$. This relation, together with (96), finally leads to the required parameterization of $Q_{h}$ in the form

$$
\alpha g \frac{h}{2} Q_{h}=\left(1-r_{1}\right) c\left|\tau_{0}\right|^{3 / 2}-\alpha g \frac{h}{2}\left[\left(1-\frac{r_{2}}{2}\right) Q_{0}+\frac{r_{2}}{2}\left|Q_{0}\right|\right] .
$$

The recipe to get $T_{0}(t)$ and $h(t)$ is now as follows: $\tau_{0}$ and $Q_{0}$ are specified forcing terms and if $Q_{h}$ from (98) is positive the two functions $T_{0}(t)$ and $h(t)$ follow from (93) and (94). If $Q_{h}$ from (98) becomes negative we determine $h$ from (98) by setting the rhs to zero, and $T_{0}$ from (93) with $Q_{h}=0$. Though this recipe appears rather technical the physics of the model should be quite clear and there are only two adjustable parameters, $\left(1-r_{1}\right) c$ and $r_{2}$. Typical parameter values are $c=1, r_{1}=0.1, r_{2}=0.9, \tau_{0}=10^{-4} \mathrm{~m}^{2} \mathrm{~s}^{-2}, Q_{0}=2.5 \times 10^{-5} \mathrm{Kms}^{-1}, \alpha=10^{-4} \mathrm{~K}^{-1}$.

Further reading: Frankignoul and Hasselmann (1977), Kraus (1977), Lemke (1986)

\subsection{ENSO models}

The strongest known variability of the climate system is the El Niño/Southern Oscillation (ENSO) phenomenon. It has time scales of months to a few years and, though being centered in the tropical Pacific, there are dramatic effects all over the globe in the climate variables as temperature and rainfall but also - as a consequence - in the economy of many states in the tropical belt (see e.g. Philander 1990)

El Niño is an aperiodic warming of the surface layer of the equatorial Pacific Ocean, occurring roughly every four years and lasting four some months, with largest amplitudes in sea surface temperature around Christmas on the Peruvian coast. It is closely connected to another large scale variability, the Southern Oscillation, which is a seesaw of atmospheric mass motions across half of the globe, already detected in 1920th by Sir Gilbert Walker and visible in the sealevel pressure variation between Djakarta and Tahiti. ENSO is known today as a coupled ocean-atmosphere mode of climate variability. 
4.4.1. Coupled instabilities The upper ocean wave system propagates in a surface layer above the equatorial thermocline of mean thickness $d$ (roughly 150 $\mathrm{m}$ ), residing over a deep motionless layer. The dynamics are represented by a reduced gravity model (cf. section 3.4), or equivalently, by a baroclinic wave model, adjusted to the equatorial belt,

$$
\begin{aligned}
\frac{\partial u}{\partial t}-\beta y v+\frac{\partial p}{\partial x} & =-\nu_{o} u+\tau^{x} / d \\
\frac{\partial v}{\partial t}+\beta y u+\frac{\partial p}{\partial y} & =-\nu_{o} v+\tau^{y} / d \\
\frac{\partial p}{\partial t}+c_{o}^{2}\left(\frac{\partial u}{\partial x}+\frac{\partial v}{\partial y}\right) & =-\mu_{o} p .
\end{aligned}
$$

with $c_{o}^{2}=g^{\prime} d$. It is driven by a wind stress $\left(\tau^{x}, \tau^{y}\right)$ and damped by a simple linear damping law with coefficients $\nu_{o}$ and $\mu_{o}$. The pressure is here only an anomaly, related to the interface displacement anomaly $\xi-d$ by $p=g^{\prime}(\xi-d)$. The atmosphere is a corresponding wave system in a two-layer troposphere in a state of the first baroclinic mode described by amplitudes $U, V, P$ and governed by

$$
\begin{aligned}
\frac{\partial U}{\partial t}-\beta y V+\frac{\partial P}{\partial x} & =-\nu_{a} U \\
\frac{\partial V}{\partial t}+\beta y U+\frac{\partial P}{\partial y} & =-\nu_{a} V \\
\frac{\partial P}{\partial t}+c_{a}^{2}\left(\frac{\partial U}{\partial x}+\frac{\partial V}{\partial y}\right) & =-\mu_{a} P-Q .
\end{aligned}
$$

Here $c_{a}^{2}=N^{2} D^{2}$, where $D$ is a vertical scale of the model. It is forced by anomalous heating $Q$ (evaporation or precipitation). The simplest coupling assumes that the wind stress is linearly related to the atmospheric wind and the heating to the oceanic interface anomaly, thus

$$
\tau^{x} / d=\gamma U \quad \tau^{y} / d=\gamma V \quad Q=\alpha(\xi-d)
$$

with parameters $\alpha$ and $\gamma$. The system can be solved analytically (various simplifications can be made, such as filtering of gravity waves, see previous section; the atmospheric system can even be considered stationary because it adjusts on a much faster time scale than the ocean system). Coupled wave modes are obtained which become unstable for certain ranges of the parameters. The instability has a simple physical explanation: suppose that the oceanic interface deepens somewhere by an amount $\Delta \xi$, this will increase the anomalous heating $Q$ (equation (105)) which in turn excites a convergent low level wind field (equation (104)), leading to convergent ocean currents (equations (105) and (99) to (100)). These in 
turn further increase $\xi$ via (101), leading to a positive feedback. This instability is a fundamental ingredient of most ENSO models.

4.4.2. The ZeBiaK-Cane model of ENSO To simulate the more complex ENSO phenomenon only a few more physical accessories have to be implemented. At first it is evident that the heating $Q$ is only fairly indirectly related by the interface anomaly, it should rather depend on the sea surface saturation humidity or temperature (cf. the bulk formulae in (25)). This requires to consider the heat balance of the oceanic surface layer which is assumed to be well mixed in the vertical. Furthermore, this layer - which is active in exchanging heat and momentum with the atmosphere - is generally shallower than the upper ocean layer considered above for the wave propagation. We thus distinguish between the 'wave layer' of depth $d$ and the 'active top layer' of depth $h_{1}$ such that $d=h_{1}+h_{2}$. Zebiak and Cane (1987, henceforth ZC) thus implement an additional surface Ekman layer of depth $h_{1} \approx 50 \mathrm{~m}$ with velocities $\left(u_{E}, v_{E}\right)$, governed by frictional dynamics,

$$
\begin{aligned}
& -\beta y v_{E}=-\nu_{o} u_{E}+\tau^{x} / h_{1} \\
& +\beta y u_{E}=-\nu_{o} v_{E}+\tau^{y} / h_{1},
\end{aligned}
$$

such that $\boldsymbol{u}_{1}=\boldsymbol{u}+h_{2} \boldsymbol{u}_{E} / d, \boldsymbol{u}_{2}=\boldsymbol{u}-h_{1} \boldsymbol{u}_{E} / d$ are the velocities in the upper (active) and lower parts of the oceanic wave layer, respectively. Hence $h_{1} \boldsymbol{u}_{1}+$ $h_{2} \boldsymbol{u}_{2}=d \boldsymbol{u}$. The heat balance of the top active layer is given by

$$
\frac{\partial T}{\partial t}+\boldsymbol{u}_{1} \cdot \nabla T+\Theta\left(w_{1}\right) w_{1}\left(T-T_{s}\right)=-\epsilon_{T}(T-\bar{T}),
$$

where $T$ is the total sea surface temperature, $w_{1}=h_{1} \nabla \cdot \boldsymbol{u}_{1}$ is the upwelling velocity at the base of the top layer, $\Theta(w)$ the Heaviside function and the Newtonian cooling term on the rhs is the heating rate of the layer, it brings $T$ back to an equilibrium value $\bar{T}$ which represents the seasonal cycle. The vertical advection term mimics numerical differencing. The upwelled water has a temperature $T_{s}=(1-\vartheta) T+\vartheta T_{d}$, it is considered to be a mixture of water in the top layer and subsurface water of a temperature $T_{d}$ at the base depth $z=-d$. The latter is parameterized as a nonlinear function $T_{d}(\xi)$ of the interface displacement. ZC take $T_{d}(\xi)=T^{*} \tanh b^{*} \xi$ with constants $T^{*}$ and $b^{*}$ which take different values for positive or negative $\xi$. Finally, the heating rate $Q$ of the atmosphere in (104) - and thereby the coupling the mixed layer temperature to the wind - must be specified. It consists of heating due to local evaporation and due to low-level moisture convergence in the atmosphere. $\mathrm{ZC}$ define $Q^{*}(T)=\mu T \exp \left[\left(T-30^{\circ} \mathrm{C}\right) / 16.7^{\circ} \mathrm{C}\right]$, where $T$ is measured in degree Celsius and $\mu$ is a constant, but feed $Q=Q^{*}(T)-Q^{*}(\bar{T})$ into the atmosphere. Notice that only the atmospheric and oceanic anomalous state is given by (99) to (104). 
The seasonal cycle $\bar{T}$ is then prescribed from observations. ZC apply the filtering concept explained in section 3.1: they omit all time derivatives in the atmospheric model and filter the gravity waves for the ocean part which is then given by the wave equations (62) and (63). Also the no-flow boundary conditions explained there are used in the $\mathrm{ZC}$ model. It is obvious that running the $\mathrm{ZC}$ model requires to specify a huge set of empirical parameters and tuning to seasonal variations of the equatorial upper ocean.

Further reading: Philander (1990), McCreary and Anderson (1991), Neelin and Latif (1994), Neelin et al. (1998)

\subsection{The wind- and buoyancy-driven horizontal ocean circulation}

The first analytical models of the wind-driven ocean circulation (Stommel 1948, Munk 1950) have ignored the stratification of the fluid and nonlinearity. They assume that a wind-driven flow regime resides in a layer of uniform depth $h$ which has no communication with underlying water (alternatively a flat-bottom ocean of depth $h$ may be considered). The vertically integrated two-dimensional flow can be represented by a streamfunction of the mass transport, $\int_{-h}^{0} \boldsymbol{u} d z=\boldsymbol{k} \times \boldsymbol{\nabla} \Psi$, if the rigid lid approximation ( $w=0$ at the mean sea surface $z=0)$ is made. Integration of the horizontal momentum balances (31) and (32) and elimination of the pressure gradient term by cross-differentiation yields the Stommel-Munk model of the circulation in a homogeneous ocean,

$$
\frac{\partial}{\partial t} \nabla^{2} \Psi+\beta \frac{\partial \Psi}{\partial x}=\operatorname{curl} \tau_{0}+A_{h} \nabla^{4} \Psi-R_{b} \nabla^{2} \Psi
$$

with the appropriate conditions $\Psi=$ const at lateral boundaries. The second term on the rhs derives from lateral diffusion of momentum, the third from linear bottom friction. Only one of these processes is necessary to extract the momentum imparted by the wind stress. The solution consists of forced, damped long Rossby waves which set up a gyre circulation in a basin with a narrow frictionally controlled western boundary current (where friction and the $\beta$-term balance) and a broad recirculation in the interior (the Sverdrup regime where the $\beta$-term and wind forcing balance).

Equations for the total mass transport from top to bottom of the ocean,

$$
\boldsymbol{U}=\int_{-h}^{\zeta} \boldsymbol{u} d z
$$

are readily derived from (12) to (17) by vertical integration, 


$$
\begin{aligned}
\frac{\partial \boldsymbol{U}}{\partial t}+f \boldsymbol{k} \times \boldsymbol{U}+g(h+\zeta) \nabla \zeta & =-h \nabla p_{b}^{c l i n}-\nabla \epsilon+\boldsymbol{\tau}_{0}-R_{b} \boldsymbol{U}+A_{h} \nabla^{2} \boldsymbol{U}=\boldsymbol{F} \\
\frac{\partial \zeta}{\partial t}+\nabla \cdot \boldsymbol{U} & =E-P=X .
\end{aligned}
$$

They take the wave response of the surface elevation into account and consider topography and stratification. The bottom pressure $p_{b}=g \zeta+p_{b}^{\text {clin }}$ has been split into the surface component $g \zeta$ and the baroclinic part given by

$$
p_{b}^{c l i n}=g \int_{-h}^{0} \rho d z \quad \text { and } \quad \epsilon=g \int_{-h}^{\zeta} z \rho d z .
$$

The latter is the potential energy (referred to the surface). We have taken the same friction laws and neglected nonlinear terms from the momentum advection as above in (108). The correspondence of (110) to the wave problem in section 3.1 is obvious: we are dealing here with the gravest mode describing barotropic gravity and Rossby waves forced by wind stress $\boldsymbol{\tau}_{0}$, surface mass flux $E-P$, and the baroclinic pressure fields as given by the gradient of the baroclinic bottom pressure $p_{b}^{c l i n}$ and the potential energy $\epsilon$.

Obviously, if $h=$ const and $\zeta \ll h$ and if the mass conservation in (110) is approximated by $\nabla \cdot \boldsymbol{U}=0$ the Stommel-Munk model (108) is obtained from (110). A few other interesting equations with more elaborate physics are derived from (110):

- The topographic Stommel-Munk problem follows if an ocean with varying depth $h$ is considered. Equation (108) then takes the form

$$
\left(\frac{\partial}{\partial t}+R_{b}\right) \nabla \cdot\left(\frac{1}{h} \nabla \Psi\right)+\frac{\partial(\Psi, f / h)}{\partial(x, y)}=\boldsymbol{k} \times \nabla(\boldsymbol{\tau} / h)+A_{h} \nabla \cdot\left(\frac{1}{h} \nabla \nabla^{2} \Psi\right) .
$$

The characteristics are the $f / h$-contours (called 'geostrophic contours, they replace the $f$-contours valid for (108)). The $h$-dependence of the friction terms may be ignored since they are anyhow only parameterizations of unknown turbulent transports of vorticity (i.e. $h$ in the last term of (112) should be replaced by a constant). Solutions for simple patterns of topography can be found e.g. in Salmon (1998).

- As in case of the wave problem (43) to (45) the equations (110) of the barotropic mode may be cast into evolution equations for the vorticity $Z=\boldsymbol{k} \times \nabla \cdot \boldsymbol{U}$, the divergence $D=\nabla \cdot \boldsymbol{U}$ and the surface elevation $\zeta$, 


$$
\begin{aligned}
\frac{\partial Z}{\partial t}+f D+\beta V & =\boldsymbol{k} \times \nabla \cdot \boldsymbol{F} \\
\frac{\partial D}{\partial t}-f Z+\beta U+g \nabla(h+\zeta) \nabla \zeta & =\nabla \cdot \boldsymbol{F} \\
\frac{\partial \zeta}{\partial t}+D & =X .
\end{aligned}
$$

The Helmholtz representation of $\boldsymbol{U}$ by a potential and a streamfunction, $\boldsymbol{U}=\nabla \Phi+\boldsymbol{k} \times \nabla \Psi$, implies $Z=\nabla^{2} \Psi$ and $D=\nabla^{2} \Phi$. For a large scale flow the non-divergent part (described by $\Psi$ ) dominates but corrections by the potential flow may have to be considered. The divergence equation (114) is then approximated by $-f Z+\beta U+g \nabla h \nabla \zeta \approx 0$ (this eliminates the gravity waves), or

$$
\nabla \cdot(f \nabla \Psi)=g \nabla \cdot(h \nabla \zeta)
$$

which is the linear balance equation. The approximate solution is $\Psi \approx$ $(g h / f) \zeta$. Taking a constant $h=H$ for simplicity the vorticity equation (113) and mass conservation (115) then combine to the linear balance model

$$
\frac{\partial}{\partial t}\left(\nabla^{2}-\frac{1}{\lambda_{0}^{2}}\right) \Psi+\beta \frac{\partial \Psi}{\partial x}=\operatorname{curl} \tau_{0}+A_{h} \nabla^{4} \Psi-R_{b} \nabla^{2} \Psi
$$

Compared to (108) this vorticity balance considers the effect of the elevation of the surface (i.e. the 'rigid lid approximation' is not applied). It finds its manifestation in the stretching term $-\Psi / \lambda_{0}^{2}$ adding to the vorticity $\nabla^{2} \Psi$ and it yields the correct form of the long barotropic Rossby waves (long compared with the barotropic Rossby radius $\lambda_{0}=\sqrt{g H} / f$ ).

Another approximation of (113) to (115) neglects the change of surface elevation in (115), so that the divergence is determined by the diagnostic relation $D=X$. This is a filtering of barotropic gravity and Rossby waves which can be applied in the ocean if time scales longer than a few days are considered and if the time evolution arising from the propagation of these waves is not of interest. We arrive at

$\frac{\partial}{\partial t} \nabla^{2} \Psi+\beta \frac{\partial \Psi}{\partial x}=-f(E-P)+\operatorname{curl} \tau_{0}+A_{h} \nabla^{4} \Psi-R_{b} \nabla^{2} \Psi$

Compared to (108) the generation of barotropic vorticity by the surface mass flux $E-P$ is included. The ratio $f(E-P) /$ curl $\tau$ is fairly small 
(of order 0.01) but it is interesting that (118) was solved already in 1933 by Goldsbrough in his study of ocean currents forced by evaporation and precipitation (Goldsbrough 1933) and also by wind (Goldsbrough 1934), well before the dynamical regimes of the wind-forced ocean circulation in the Stommel-Munk model (108) was rediscovered in the oceanographic community (see also Stommel 1984).

- The above described theories of the vertically integrated circulation have neglected the effect of the baroclinic pressure forces in (110) altogether. The effect can be investigated by a simple barotropic-baroclinic interaction model (cf. Olbers and Wolff, 2000). Let us assume for simplicity that the balance of total mass (the second equation in (110)) is approximated by the rigid lid form $\nabla \cdot \boldsymbol{U}=0$. We also abandon lateral diffusion of momentum for simplicity. Taking the curl of the momentum balance (110) yields

$$
\left(\frac{\partial}{\partial t}+R_{b}\right) \nabla \cdot\left(\frac{1}{h} \nabla \Psi\right)+\frac{\partial(\Psi, f / h)}{\partial(x, y)}=\boldsymbol{k} \times \nabla(\boldsymbol{\tau} / h)-\frac{\partial\left(\epsilon / h^{2}, h\right)}{\partial(x, y)} .
$$

Compared to the topographic - completely wind stress forced - StommelMunk problem (112) we realize a second vorticity source stemming from the baroclinic pressure term of the baroclinic potential energy $\varepsilon$ : the last term on the rhs of (119) is called the JEBAR-term (Joint Effect of Baroclinicity and bottom Relief) or the baroclinic bottom torque. Coupling to the stratification only occurs where the bottom is not flat. Estimation of this term shows that it is of overwhelming importance compared to the wind stress curl unless $\epsilon$-contours follow closely the contours of $h$.

The bottom torque can be considered as a prescribed source in (119) but, in fact, it is determined from the thermohaline balances (15) to (17) of the full dynamical problem of the ocean circulation. We expand the density about a reference field, described by the Brunt-Väisälä frequency $N(z)$, as in section 3.1, and assume $N$ to be constant. Projecting (15) to (17) on the baroclinic potential energy and retaining in the advection only the barotropic flow we get a coupled set of equations for the streamfunction $\Psi$ and the potential energy $\epsilon=\epsilon^{\prime}-(1 / 3) N^{2} h^{3}$, given by (119) and

$$
\frac{\partial \epsilon^{\prime}}{\partial t}+h \frac{\partial\left(\Psi, \epsilon^{\prime} / h^{2}\right)}{\partial(x, y)}-\frac{1}{3} h N^{2} \frac{\partial(\Psi, h)}{\partial(x, y)}=Q+K_{h} \nabla^{2} \epsilon^{\prime} .
$$

According to this simplified balance, potential energy is provided by a surface buoyancy flux $Q$ and advected by the barotropic flow (the second and the third term on the lhs, the latter is the vertical advection of the reference state). We have additionally included lateral diffusion of density in the last term on the rhs. Whereas the restricted barotropic problem (112) contains only barotropic Rossby waves, the coupled barotropic-baroclinic 
problem (119) and (120) additionally contains a baroclinic Rossby wave. It also allows forcing of the circulation by fluxes of heat and freshwater (combining to $Q$ ) at the ocean surface. Notice that the bottom torque only arises from the perturbation potential energy $\epsilon^{\prime}$, i.e. $\varepsilon$ in (119) may be replaced by $\varepsilon^{\prime}$. Notice also that the consideration of the stratification - in concert with varying topography - makes the determination of the integrated circulation a nonlinear problem.

- The generalization (108) to nonlinear advection is obvious. Equations (110) are in fact Laplace tidal equations if the forcing is replaced by the tidal forcing (the momentum is forced by the tidal potential arising from attraction of the ocean water by moon, sun and planets). Laplace derived the equations for a homogeneous ocean where they read with full nonlinearities

$$
\begin{array}{r}
\frac{\partial \boldsymbol{u}}{\partial t}+\boldsymbol{u} \cdot \nabla \boldsymbol{u}+f \boldsymbol{k} \times \boldsymbol{u}+g \nabla \zeta=\boldsymbol{F} / h \\
\frac{\partial \zeta}{\partial t}+\nabla \cdot[(h+\zeta) \boldsymbol{u}]=X .
\end{array}
$$

To derive (121) it must be assumed that $\boldsymbol{u}=\boldsymbol{U} /(h+\zeta)$ is vertically constant.

- The nonlinear Stommel-Munk problem is derived from the above equations for $h=$ const, $\zeta \ll h$ and neglecting the tendency and surface flux in the mass balance, i.e. $\nabla \cdot \boldsymbol{u}=0$ and thus $\boldsymbol{u}=\boldsymbol{k} \times \nabla \varphi$ with a streamfunction $\varphi$. Then

$$
\frac{\partial}{\partial t} \nabla^{2} \varphi+\frac{\partial\left(\varphi, \nabla^{2} \varphi+f\right)}{\partial(x, y)}=\operatorname{curl} \tau_{0} / h+A_{h} \nabla^{4} \varphi-R_{b} \nabla^{2} \varphi
$$

- The nonlinear balance equations are obtained by forming vorticity and divergence of (121). Neglecting the rate of change of the divergence, as above for the linear problem, one finds three coupled non-linear equations,

$$
\begin{array}{r}
\left(\frac{\partial}{\partial t}+\nabla^{2} \phi+\boldsymbol{u} \cdot \nabla\right)\left(\nabla^{2} \psi+f\right)=\operatorname{curl} \boldsymbol{F} / h \\
\nabla \cdot(f \nabla \psi)+2 \frac{\partial(\partial \psi / \partial x, \partial \psi / \partial y)}{\partial(x, y)}=g \nabla^{2} \zeta \\
\left(\frac{\partial}{\partial t}+\boldsymbol{u} \cdot \nabla\right) \zeta+h \nabla^{2} \phi=X
\end{array}
$$


where $\boldsymbol{u}=\boldsymbol{k} \times \nabla \psi+\nabla \phi$. These equations were first discussed by Bolin (1955) and Charney (1955). Solutions - even numerical - are rather difficult to obtain. The nonlinear balance equation have recently regained some interest in oceanographic applications (Gent and McWilliams 1983a,b).

Notice that some of these linear two-dimensional circulation problems are easily reduced to one spatial dimension, namely those with constant coefficients. Consider the circulation in a rectangular box, driven by a wind stress that is sinusoidal in the meridional direction, curl $\boldsymbol{\tau}_{0}=T(x, t) \operatorname{Sin}(y)$, where $\operatorname{Sin}(y)$ is an eigenfunction of $\partial^{2} / \partial y^{2}$ (with eigenvalue $-\ell^{2}$ ), which vanishes on the southern and northern boundaries of the box ocean. With $\Psi(x, y, t)=P(x, t) \operatorname{Sin}(y)$, the Stommel-Munk problem (108) reduces to

$$
\frac{\partial}{\partial t}\left(\frac{\partial^{2}}{\partial x^{2}}-\ell^{2}\right) P+\beta \frac{\partial P}{\partial x}=T+A_{h}\left(\frac{\partial^{4}}{\partial x^{4}}+\ell^{4}\right) P-R_{b}\left(\frac{\partial^{2}}{\partial x^{2}}-\ell^{2}\right) P
$$

with boundary conditions $P=0$ on the western and eastern boundaries of the box. A similar reduction is possible for (117) and (118).

Further reading: Pedlosky (1986), Salmon (1998), Frankignoul et al. (1997)

\subsection{The thermohaline-driven meridional ocean circulation}

The vertical integral of the equations of motion emphasizes the wind-driven part of the ocean circulation. The effects of stratification appear as forcing in the equations of the horizontal mass transport. A complementary view is gained from zonal integration. Marotzke et al. (1988) and Stocker and Wright (1991) and numerous authors thereafter have used this framework to study the thermohaline (or overturning) circulation in a simplified model of ocean circulation. Zonally integrated diagnostics and models are quite common in atmospheric studies (cf. next section), for the investigation of the oceanic overturning they recently got attention, mainly because they are considerable less expensive than full 3-d simulations.

We take the planetary geostrophic equations (68) to (72), supplemented by vertical friction to couple directly to the wind forcing at the ocean surface. For simplicity we stick to the thermohaline density equation (72) though a more complete model should use the balances of heat and salt separately and apply the complete equation of state. We consider a closed ocean basin with no islands (an idealized Atlantic Ocean closed by a southern coast) of zonal width $\Delta \lambda(\phi)$ at latitude $\phi$ and define zonal averages of all fields, e.g. $\tilde{\rho}(\phi, z)=(1 / \Delta \lambda) \int \rho(\lambda, \phi, z) d \lambda$. The zonally averaged equations become 


$$
\begin{aligned}
-f \tilde{v} & =-\frac{1}{a \cos \phi} \frac{\Delta p}{\Delta \lambda}+A_{v} \tilde{u}_{z z} \\
f \tilde{u} & =-\frac{1}{a} \tilde{p}_{\phi}+A_{v} \tilde{v}_{z z} \\
\tilde{p}_{z} & =-g \tilde{\rho} \\
\frac{1}{a \cos \phi}(\tilde{v} \cos \phi)_{\phi}+\tilde{w}_{z} & =0 \\
\tilde{\rho}_{t}+\frac{\tilde{v}}{a} \tilde{\rho}_{\phi}+\tilde{w} \tilde{\rho}_{z} & =\left(K_{v} \tilde{\rho}_{z}\right)_{z}+\frac{1}{a^{2} \cos \phi}\left(K_{h} \tilde{\rho}_{\phi} \cos \phi\right)_{\phi}+q .
\end{aligned}
$$

The meaning of $q$ is outlined below. The dynamics of this model may be condensed to two coupled nonlinear differential equations for the density $\tilde{\rho}$ and the meridional overturning streamfunction $\Lambda(\phi, z)$, which is introduced on the basis of (128),

$$
\tilde{v}=-\frac{1}{\cos \phi} \frac{\partial \Lambda}{\partial z} \quad \tilde{w}=\frac{1}{a \cos \phi} \frac{\partial \Lambda}{\partial \phi} .
$$

One finds

$$
\begin{aligned}
f^{2} \Lambda_{z}+A_{v}^{2} \Lambda_{z z z z z} & =\frac{g}{a} A_{v} \cos \phi \tilde{\rho}_{z \phi}-\frac{f \Delta p}{a \Delta \lambda} \\
\tilde{\rho}_{t}+\frac{1}{a \cos \phi} \frac{\partial(\Lambda, \tilde{\rho})}{\partial(\phi, z)} & =\frac{1}{a^{2} \cos \phi}\left(K_{h} \tilde{\rho}_{\phi} \cos \phi\right)_{\phi}+\left(K_{v} \tilde{\rho}_{z}\right)_{z}+q .
\end{aligned}
$$

This set of equations is not closed. At first, the term $q$ on the rhs of the thermohaline balance contains the divergence of Reynolds-type fluxes $\int(v-\tilde{v})(\rho-\tilde{\rho}) d \lambda$. At the present stage of the 2-d thermohaline models these fluxes are ignored. Secondly, the pressure difference $\Delta p(\phi, z)$ is not known, it cannot be ignored and must be parameterized in terms of the resolved (zonally averaged) fields. Marotzke et al. (1988) effectively replace (131) by a modified version

$$
A_{v}^{*} \Lambda_{z z z z}=\frac{g}{a} \cos \phi \tilde{\rho}_{\phi},
$$

with rescaled friction coefficient $A_{v}^{*}$ of order $A_{v}^{*} \sim A_{v}\left(1+\left(f h^{2} / A_{v}\right)^{2}\right)$. The equation postulated a linear relation $a \cos \phi A_{v}^{*} \tilde{v}_{z z}=\tilde{p}_{\phi}$. Stocker and Wright (1991) use the parameterization

$$
\Delta p=g \epsilon_{0} \sin 2 \phi \int_{z}^{0} \rho_{\phi} d z
$$


with $\epsilon_{0}=0.3$, derived from experiments with full 3-d dynamics.

The system (131) and (132) needs boundary conditions at the top, the bottom, and the northern and southern restrictions. The kinematic condition of zero normal velocity is $\Lambda=$ const on all boundaries. For a 'box' ocean of constant depth $H$ the thermohaline balance is supplemented by flux conditions $K_{h} \tilde{\rho}_{\phi}=0$ at lateral (north and south) boundaries, and $K_{v} \tilde{\rho}_{z}=Q_{\rho}$ at $z=0$ and $K_{v} \tilde{\rho}_{z}=0$ at $z=-H$, where $Q_{\rho}$ is the density flux established by heat and freshwater transfer at the ocean surface. Finally, frictional boundary conditions regulate the transfer of stresses across the models interfaces at top and bottom. Various possible combinations of stress or no-slip conditions can be used, a typical example is $A_{v} \tilde{v}_{z}=0$ leading to $\Lambda_{z z}=0$ at $z=0,-H, A_{v} \tilde{u}_{z}=\tau^{\lambda}$ at $z=0, A_{v} \tilde{u}_{z}=0$ at $z=-H$, where the vertical shear translates into $\tilde{u}_{z}=g \tilde{\rho}_{\phi} /(a f)-A_{v} \Lambda_{z z z} /(f \cos \phi)$, and $\tau^{\lambda}$ is the zonal wind stress. Alternatively, the no-slip condition $\Lambda_{z}=0$ may be taken at the bottom. The condition on the zonal stress cannot be incorporated into Marotzke's model.

It should be mentioned that the model might yield an unstable density stratification, in the sense that heavier water resides on top of lighter water. This is a consequence of the hydrostatic approximation which has canceled the vertical acceleration as natural reaction to such a situation. For practical applications, ocean models implant a very strong vertical mixing of density (heat and salt) at corresponding locations. These are hidden in the term $q$ in the thermohaline balance.

Further reading: Broecker (1991), Rahmstorf et al. (1996)

\subsection{Symmetric circulation models of the atmosphere}

With slight simplifications the model also describes an important aspect of the atmospheric circulation. Here we consider averaging around complete latitude circles so that $\Delta p=0$ and the associated parameterization problem does not exist. While atmospheric fields are far from being zonally symmetric (i.e. independent on longitude; actually the oceanic circulation is even more 'asymmetric') the concept of a symmetric atmospheric state has a long history (see e.g. Lorenz (1967) for a review) and even today many aspects of data interpretation uses zonal averaging (cf. Peixoto and Oort 1992). Various attempts have been made to construct a corresponding symmetric model (e.g. Schneider and Lindzen 1977, Held and Hou 1980). For the atmospheric case, equation (129) should be replaced by the heat balance (16), considered in the above investigations with simplified heating in form of restoring to a prescribed climatology $\theta_{e}(\phi, z)$ of the radiative equilibrium temperature distribution. The heat balance then reads

$$
\tilde{\theta}_{t}+\frac{\tilde{v}}{a} \tilde{\theta}_{\phi}+\tilde{w} \tilde{\theta}_{z}=\left(K_{v} \tilde{\theta}_{z}\right)_{z}+\frac{1}{a^{2} \cos \phi}\left(K_{h} \tilde{\theta}_{\phi} \cos \phi\right)_{\phi}-\frac{\tilde{\theta}-\theta_{e}}{t_{R}}
$$

where $\tilde{\theta}$ refers to the potential temperature. A simplified form of $\theta_{e}$ is given by 


$$
\theta_{e}(\phi, z)=\theta_{0}\left[1-\Delta_{h}\left(\frac{1}{3}+\frac{2}{3} P_{2}(\sin \phi)\right)+\Delta_{v}(z-H / 2)\right]
$$

where $\Delta_{h}$ is the relative temperature drop from equator to pole, $\Delta_{v}$ the drop from the height $H$ to the ground, and $P_{2}$ the Legendre polynomial of second degree. The equation of state must be used to relate $\tilde{\rho}$ and $\tilde{\theta}$, also here the system is simplified using $\tilde{\rho}=-\tilde{\theta} / \theta_{0}$ (remember that $\rho$ is the dimensionless Boussinesq variable). In contrast to the oceanic case where forcing by the thermohaline boundary conditions spreads its effect in the interior by advection and diffusion, the dominating balance in (135) is between the local heating and advection. The most simple version even omits the meridional advection and linearizes the vertical term, so that we obtain

$$
\begin{aligned}
f^{2} \Lambda_{z}+A_{v}^{2} \Lambda_{z z z z z} & =-\frac{g}{a \theta_{0}} A_{v} \cos \phi \tilde{\theta}_{z \phi} \\
\tilde{\theta}_{t}+\frac{1}{a \cos \phi} \Lambda_{\phi} \Theta_{z} & =-\frac{\tilde{\theta}-\theta_{c}}{t_{R}},
\end{aligned}
$$

with constant and prescribed $\Theta_{z}$.

Further reading: Lindzen (1990), James (1994)

\section{Low-order models}

The models considered in the previous sections are described by partial differential equations, some cases are even nonlinear. Analytical solutions are known only for the most simple, fairly restrictive conditions. In some cases even numerical solutions are difficult to obtain. To gain insight into the behavior of the climate system on a more qualitative level low-order models are developed. They resolve the spatial structures in a truncated aspect but allow nonlinearities to be considered in detail. The construction is simple: the spatial structure of the fields is represented by a set of prescribed structure functions with time dependent amplitudes. Projection of the evolution equations then yields a set of coupled ordinary differential equations for the amplitudes. Proper selection of these spatial functions is of course the most delicate and important problem in the construction of a low-order model. Most of such models apply to atmospheric systems. The oceans are embedded in rather irregular basins and even simple box-type oceans develop dynamically important boundary layers (as the Gulf Stream) which defies representation by simple structure functions. Nevertheless, we have some oceanic low-order models as well.

An early example of a nonlinear low-order model is found in Lorenz (1960) where the philosophy and truncation method is explained for a barotropic QG flow for atmospheric conditions. The expansion of the streamfunction $\psi$ into a complete set of orthogonal function is truncated to an interacting triad 


$$
\psi=-\left(A / \ell^{2}\right) \cos \ell y-\left(F / k^{2}\right) \cos k x-2 G /\left(k^{2}+\ell^{2}\right) \sin \ell y \sin k x
$$

with zonal and meridional wavenumbers $k$ and $\ell$. The flow consists of mean zonal and meridional components with amplitudes $A$ and $F$, respectively, and a wave mode with amplitude $G$. The system is governed by

$$
\begin{aligned}
\dot{A} & =-\left(\frac{1}{k^{2}}-\frac{1}{k^{2}+\ell^{2}}\right) k \ell F G-\mu A+X \\
\dot{F} & =\left(\frac{1}{\ell^{2}}-\frac{1}{k^{2}+\ell^{2}}\right) k \ell A G-\mu F+Y \\
\dot{G} & =-\frac{1}{2}\left(\frac{1}{k^{2}}-\frac{1}{\ell^{2}}\right) k \ell A F-\mu G+Z
\end{aligned}
$$

with forcing $X, Y, Z$ and dissipation by linear friction included. If these are absent the energy $\left(A^{2} / \ell^{2}+F^{2} / k^{2}+2 G^{2} /\left(k^{2}+\ell^{2}\right)\right) / 4$ and the enstrophy $\left(A^{2}+F^{2}+2 G^{2}\right) / 2$ of the system are conserved so an analytical solution of the equations (in terms of elliptic functions) is possible. Periodic solutions arise entirely due to nonlinear interaction of the triad. Notice that only the aspect ratio $\alpha=k / \ell$ of the wave vector is relevant. A stochastic variant with white noise $X, Y, Z$ is discussed in Egger (1999). Typical parameter values are $\alpha=0.9, \mu=10^{-6} s^{-1}$ and white noise with $<X^{2}>^{1 / 2}=10^{-10} s^{-2}$.

\subsection{Benard convection}

A fluid which is heated from below develops convective motions. The linear stage of instability is treated in the classical monograph of Chandrasekhar (1961), a low-order model for the nonlinear evolution is Lorenz' famous chaotic attractor (Lorenz 1963).

Consider a layer of vertical extent $H$ where the temperature at top and bottom is held fixed, $\theta(x, y, z=0, t)=\theta_{0}+\Delta \theta$ and $\theta(x, y, z=H, t)=\theta_{0}$. We assume for simplicity invariance in the $y$-direction and introduce a streamfunction $\Psi(x, z, t)$ with $u=-\partial \Psi / \partial z$ and $w=\partial \Psi / \partial x$ and the temperature perturbation $\Theta(x, z, t)$ about a linear profile with amplitude $\Delta \theta$,

$$
\theta(x, z, t)=\theta_{0}+\Delta \theta\left(1-\frac{z}{H}\right)+\Theta(x, z, t)
$$

Eliminating the pressure from the $x$ - and $z$-component of (5) (without rotation), assuming a linear equation of state, $\varrho=\varrho_{0}\left(1-\alpha\left(\theta-\theta_{0}\right)\right)$, and inserting (141) into (8) we get 


$$
\begin{aligned}
\frac{\partial}{\partial t} \nabla^{2} \Psi & =-\frac{\partial\left(\Psi, \nabla^{2} \Psi\right)}{\partial(x, z)}+g \alpha \frac{\partial \Theta}{\partial x}+\nu \nabla^{4} \Psi \\
\frac{\partial}{\partial t} \Theta & =-\frac{\partial(\Psi, \Theta)}{\partial(x, z)}+\frac{\Delta \theta}{H} \frac{\partial \Psi}{\partial x}+\kappa \nabla^{2} \Theta,
\end{aligned}
$$

where $\alpha$ is the thermal expansion coefficient, $\nu$ is the kinematic viscosity and $\kappa$ the thermal conductivity. Furthermore, $\nabla$ denotes here the $(x, z)$-gradient.

A low-order model of these equations was proposed by Lorenz (1963), it became an icon of chaotic behavior. The Lorenz equations are found by taking boundary conditions $\Theta=0, \Psi=0, \nabla^{2} \Psi=0$ at $z=0, H$ and using the truncated representation of $\Theta$ and $\Psi$ by three modes,

$$
\begin{aligned}
\frac{v}{\kappa\left(1+v^{2}\right)} \Psi & =\sqrt{2} X(t) \sin \left(\frac{\pi v}{H} x\right) \sin \left(\frac{\pi}{H} z\right) \\
\frac{\pi \mathrm{Ra}}{\mathrm{Ra}_{c} \Delta T} \Theta & =\sqrt{2} Y(t) \cos \left(\frac{\pi v}{H} x\right) \sin \left(\frac{\pi}{H} z\right)-Z(t) \sin \left(\frac{2 \pi}{H} z\right),
\end{aligned}
$$

with amplitudes $X, Y, Z$. Here $v$ is the aspect ratio of the roles and a Rayleigh number $\mathrm{Ra}=g \alpha H^{3} \Delta \theta /(\kappa \nu)$ is the introduced with critical value $\mathrm{Ra}_{c}=\pi^{4} v^{-2}(1+$ $\left.v^{2}\right)^{3}$ (this value controls the linear stability problem, see Chandrasekhar 1961). Introducing (143) into (142) one finds (the original Lorenz model has $F=0$ )

$$
\begin{aligned}
\dot{X} & =-\operatorname{Pr} X+\operatorname{Pr} Y+F \cos \vartheta \\
\dot{Y} & =-X Z+r X-Y+F \sin \vartheta \\
\dot{Z} & =X Y-b Z .
\end{aligned}
$$

The derivative is with respect to the scaled time $\pi^{2} H^{-2}\left(1+v^{2}\right) \kappa t$, dimensionless control parameters are the Prandtl number $\operatorname{Pr}=\nu / \kappa$, a geometric factor $b=$ $4\left(1+v^{2}\right)^{-1}$ and $r=\mathrm{Ra} / \mathrm{Ra}_{c} \propto \Delta \theta$ as measure of the heating. Lorenz investigated the system for $\operatorname{Pr}=10, b=8 / 3$ and positive $r$. Palmer (1998) considers a forced version of the Lorenz model (with $F \neq 0$ and various values of $\vartheta$ ), reviving the notion of the 'index cycles' of the large-scale atmospheric circulation as result of a chaotic evolutionary process. The index cycle is the irregular switching of the zonal flow between quasisteady regimes with strong and more zonal conditions and weak and less zonal (more wavy) conditions (see also section 5.4). 


\subsection{A truncated model of the wind-driven ocean circulation}

The same year that Edward Lorenz' chaotic attractor was published George Veronis applied the truncation technique to an oceanic circulation problem, the winddriven barotropic circulation in a rectangular shaped basin (Veronis 1963). The system is governed by the Stommel-Munk model (108), for simplicity with $A_{h}=0$. A square ocean box with depth $H$ and lateral size $L$ in the domain $0 \leq x \leq \pi, 0 \leq$ $y \leq \pi$ is considered. The coordinates are scaled by $L$ and time by $1 /(L \beta)$. The ocean is forced by a wind stress with curl $\boldsymbol{\tau}_{0}=-(W / L) \sin x \sin y$ and the response is represented by the truncated scaled streamfunction

$$
\begin{aligned}
\psi= & \frac{20 H^{2} \beta^{3} L^{3}}{9 W^{2}}[A \sin x \sin y+ \\
& +B \sin 2 x \sin y+C \sin x \sin 2 y+D \sin 2 x \sin 2 y] .
\end{aligned}
$$

A particular problem is the projection of the $\beta$-term: to meet the boundary condition the streamfunction must consist of sine-terms and then all terms in the vorticity balance are sine-terms with exception of the $\beta$-term which is a cosineterm. Veronis arrives at

$$
\begin{aligned}
\dot{A} & =-\frac{4}{3 \pi} B-\epsilon A+\frac{9}{40} \mathrm{Ro} \\
\dot{B} & =\frac{8}{15 \pi} A+A C-\epsilon B \\
\dot{C} & =-\frac{8}{15 \pi} D-A B-\epsilon C \\
\dot{D} & =\frac{1}{3 \pi} C-\epsilon D,
\end{aligned}
$$

where $\epsilon=R_{b} /(\beta L)$ and $\operatorname{Ro}=W /\left(\beta^{2} H L^{3}\right)$ are the nondimensional friction coefficient and wind stress amplitude, respectively. The $\beta$-term is found in the first terms on the rhs (leading to a linear oscillatory behavior), the other terms are readily identified as derived from the nonlinear and friction terms. There may be three steady state solutions, one corresponding (for small friction) to the familiar Sverdrup balance where the $\beta$-term and the wind curl balance in (146), $B \approx 27 \pi \mathrm{Ro} / 160$, and all other coefficients are small. If friction is small and the Rossby number is sufficiently large (strong wind stress), Ro $>0.32$, a frictionally controlled solution is possible where $A \approx 9 \mathrm{Ro} /(40 \epsilon)$. If $\epsilon>0.3$ only one solution exists regardless of the value of Ro. Not all solutions are stable, however: if there is only one steady solution it is stable, if there are three only the one with maximum $A$ is stable. The time dependent system has damped oscillating solutions (settling towards the Sverdrup balance) but also very complicated limit cycles (e.g. for $\epsilon=0.01$, Ro $=0.3)$. 


\subsection{The low frequency atmospheric circulation}

Any time series of atmospheric data shows variability, no matter what frequencies are resolved. In fact, the power spectra of atmospheric variables are red which means that amplitudes of fluctuations increase with increasing period. A wide range of processes is responsible for this irregular and aperiodic behavior, they overlap and interact in the frequency domain and therefore it is difficult to extract signatures of specific processes from data. A major part of the climate signals derive from the interaction of ocean and atmosphere (as e.g. ENSO, cf. section 4.4 and 5.6), others derive from the internal nonlinearity in the atmospheric dynamics

alone. Prominent processes are wave-mean flow and wave-wave interactions and the coupling of the flow to the orography of the planet. Examples of low-order models of these features are presented in the next two sections.

Besides the Lorenz attractor another low-order model with chaotic properties was introduced by Lorenz (1984) to serve as an extremely simple analogue of the global atmospheric circulation. The model is defined by three interacting quantities: the zonal flow $X$ represents the intensity of the mid-latitude westerly wind current (or, by geostrophy, the meridional temperature gradient) in the northern and southern hemisphere, and a wave component exists with $Y$ and $Z$ representing the cosine and sine phases of a chain of vortices superimposed on the zonal flow. The horizontal and vertical structures of the zonal flow and the wave are specified, the zonal flow may only vary in intensity and the wave in longitude and intensity. Relative to the zonal flow, the wave variables are scaled so that $X^{2}+Y^{2}+Z^{2}$ is the total scaled energy (kinetic plus potential plus internal). Lorenz considers the dynamical system

$$
\begin{aligned}
\dot{X} & =-\left(Y^{2}+Z^{2}\right)-a(X-F) \\
\dot{Y} & =-b X Z+X Y-Y+G \\
\dot{Z} & =b X Y+X Z-Z
\end{aligned}
$$

The system bears similarity with the Lorenz attractor (144) (as many other loworder systems derived from fluid mechanics) but additional terms appear. The vortices are linearly damped by viscous and thermal processes, the damping time defines the time unit and $a<1$ is a Prandtl number. The terms $X Y$ and $X Z$ in (151) and (152) represent the amplification of the wave by interaction with the zonal flow. This occurs at the expense of the westerly current: the wave transports heat poleward, thus reducing the temperature gradient, at a rate proportional to the square of the amplitudes, as indicated by the term $-\left(Y^{2}+Z^{2}\right)$ in $(150)$. The total energy is not altered by this process. The terms $-b X Z$ and $b X Y$ represent the westward (if $X>0$ ) displacement of the wave by the zonal current, and $b>1$ allows the displacement to overcome the amplification. The zonal flow is driven by the external force $a F$ which is proportional to the contrast between solar heating at low and high latitudes. A secondary forcing $G$ affects the wave, it mimics the 
contrasting thermal properties of the underlying surface of zonally alternating oceans and continents. The model may be derived from the equations of motion by extreme truncation along similar routes as demonstrated above for the Lorenz attractor.

When $G=0$ and $F<1$, the system has a single steady solution $X=$ $F, Y=Z=0$, representing a steady Hadley circulation. This zonal flow becomes unstable for $F>1$, forming steadily progressing vortices. For $G>0$ the system clearly shows chaotic behavior (Lorenz considers $a=1 / 4, b=4, F=8$ and $G=$ $O(1)$ ). Long integrations (see e.g. James 1994) reveals unsteadiness, even on long timescales of tens of years, with a typical red-noise spectrum.

One fairly complex but still handy low-order model was recently investigated by Kurgansky et al. (1996). It includes wave-mean flow interaction and orographic forcing. The problem is formulated in spherical coordinates, all quantities are scaled by taking the earth's radius $a$ as unit length and the inverse of the earth's rotation rate $\Omega$ as unit of time,

$$
\begin{array}{r}
\frac{\partial}{\partial t}\left(\nabla^{2} \psi-\psi / L^{2}\right)+\boldsymbol{u} \cdot \nabla\left(k \nabla^{2} \psi+2 \sin \phi+H / L^{2}\right)+L^{-2} \frac{\partial \chi}{\partial t}= \\
=L^{-2} \boldsymbol{k} \times \nabla \chi \cdot \nabla H+\nu \nabla^{2}(\chi-\psi) \\
\frac{\partial \chi}{\partial t}+\boldsymbol{u} \cdot \nabla \chi-\varepsilon \frac{\partial \psi}{\partial t}=-\varepsilon \boldsymbol{u} \cdot \nabla H+\kappa\left(\chi^{*}-\chi\right) .
\end{array}
$$

Here $H=g h /\left(\sqrt{2} a^{2} \Omega^{2}\right)$ is the scaled topography height, $L=\lambda_{1} / a$ is the scaled baroclinic Rossby radius, $\varepsilon=R /\left(R+c_{p}\right)=2 / 9$ where $R$ is the gas constant of dry air and $c_{p}$ the specific heat capacity, $\nu$ and $\kappa$ are scaled Ekman and Newtonian damping coefficients and $\kappa \chi^{*}$ the scaled heating rate. The coefficient $k=4 / 3$ is introduced to improve the model's vertical representation. The state variables $\psi$ and $\chi$ are scaled as well, they represent the streamfunction and the vertically averaged temperature field. Hence $\boldsymbol{u}=\boldsymbol{k} \times \nabla \psi$.

The equations are derived from the basic equations of motion by vertical averaging and assuming only slight deviations from a barotropic (vertically constant) state. Horizontal inhomogeneities of temperature are accounted for, and in this respect the above equations generalize the barotropic models considered in section 4.5 and the quasigeostrophic models considered in the sections 3.2 and 3.4. For more details we refer to Kurgansky et al. (1996). Basically, (153) is the balance of potential vorticity and (154) is the balance of heat. The effect of the topography on the flow is seen in the terms involving $H$ ('orographic forcing terms'). Notice also the correspondence to the Charney-DeVore model discussed below in section 5.4 .

The model may be taken as a coupled set for $\psi$ and $\chi$ in the two-dimensional domain of the sphere, with specified thermal forcing $\kappa \chi^{*}(\phi, \lambda, t)$. Kurgansky et al. (1996) reduce the degrees of freedom by constructing a low-order model, based on the representation 


$$
\begin{aligned}
& \psi=-\alpha(t) \mu+F(t) P_{N}^{0}(\mu)+A(t) P_{n}^{m}(\mu) \sin m \lambda+U(t) P_{n}^{m}(\mu) \cos m \lambda \\
& \chi=-\beta(t) \mu+G(t) P_{N}^{0}(\mu)+B(t) P_{n}^{m}(\mu) \cos m \lambda+V(t) P_{n}^{m}(\mu) \sin m \lambda,
\end{aligned}
$$

with $\mu=\sin \phi$ and $P_{n}^{m}$ denoting associated Legendre functions. Furthermore, the topography and the thermal forcing are specified as

$$
H=H_{0} P_{n}^{m}(\mu) \sin m \lambda \quad \chi^{*}=-\chi_{0}(t) \mu,
$$

where the amplitude $\chi_{0}(t)$ describes a seasonal cycle. The system is thus reduced to a zonal flow represented by $(\alpha, \beta)$ and $(F, G)$ and a wave represented by $(A, B, U, V)$. It is governed by eight coupled differential equations for these amplitudes. We refer to Kurgansky et al. (1996) because they are rather lengthy. In their experiments they adopt $m=2, n=5$ and $N=3$.

The model shows a rich low-frequency time variability, with and without seasonal forcing. Fluctuations are predominantly caused by interaction of the orographically excited standing wave and the zonal mean flow. Spectra are red up to periods of decades and chaotic behavior shows up as well.

A simplified version is obtained if the zonal contributions to $\psi$ and $\chi$, described by the amplitudes $\alpha$ and $\beta$, are considered as given constants, and orographic and thermal forcing is omitted. The model then represents the response of the wave system to the coupling of the wave to the mean flow and and wave-wave interaction. The six remaining amplitudes follow from

$$
\begin{aligned}
\dot{F} & =-\frac{1}{2} \Pi(U V-A B) \\
\dot{G} & =-\frac{1}{2} \Xi(U V-A B) \\
\dot{A} & =-\Gamma U+\Delta B-\Pi(B F-U G) \\
\dot{U} & =\Gamma A-\Delta V+\Pi(V F-A G) \\
\dot{B} & =\Upsilon A-\Sigma V+\Xi(V F-A G) \\
\dot{V} & =-\Upsilon U+\Sigma B-\Xi(B F-U G) .
\end{aligned}
$$

Time is scaled as $\left[n(n+1)+L^{-2}(1-\varepsilon)\right] t$, furthermore $k=1, N=n, H_{0}=$ 0 , and the following abbreviations are made: $\Pi=m q / L^{2}, \Delta=\alpha m / L^{2}, \Gamma=$ $m\left[2(1+\alpha)+\alpha / L^{2}-\alpha n(n+1)\right], \Upsilon=m\left[2 \varepsilon(1+\alpha)+\alpha / L^{2}+\alpha n(n+1)(1-\varepsilon)\right]$, $\Xi=m q\left[n(n+1)+L^{-2}\right]$, and $\Sigma=m \alpha\left[n(n+1)+L^{-2}\right]$, and $q$ is a triple integral 
of the Legendre functions, $q=\int\left(P_{N}^{m}\right)^{2}\left(d P_{N}^{0} / d \mu\right) d \mu$. Typical parameter values are $m=2, n=3, q=3.6, \alpha=6 \times 10^{-2}, \varepsilon=2 / 9, L^{-2}=5.7$. In this version dissipative terms have omitted as well and the system then yields self-sustained non-linear oscillations. In fact, Kurgansky et al. (1996) describe a solution of (157) in terms of elliptic functions. The model produces an interesting torus-type portrait in the phase space. The zonal thermal forcing (156), however, does not enter the equations of the six wave amplitudes and, thus, for studies of forced and dissipative problems either the complete model has to invoked or a direct thermal forcing of the wave must be considered.

Further reading: James (1994)

\subsection{Charney-DeVore models}

The state of the atmosphere in midlatitudes of the northern hemisphere shows long persisting anomalies ('Großwetterlagen') during which the movement of irregular weather variability across the Atlantic seems to be blocked. It is appealing to connect these 'Großwetterlagen' with the steady regimes of a low-order subsystem of the atmospheric dynamics and explain transitions by interaction with shorter waves simply acting as white noise. Starting with the work of Egger (1978) and Charney and DeVore (1979) the concept of multiple equilibria in a severely truncated 'low-order' image (the CdV model) of the atmospheric circulation was put forward. The observational evidence for dynamically disjunct multiple states, particularly with features of the $\mathrm{CdV}$ model, in the atmospheric circulation is however sparse (see the collection of papers in Benzi et al. 1986) and the applicability has correctly been questioned (see e.g. Tung and Rosenthal 1985).

The simplest CdV model describes a barotropic zonally unbounded flow over a sinusoidal topography in a zonal channel with quasigeostrophic dynamics. The flow is governed by the barotropic version of (65) or, in layer form, by (80). The vorticity balance of such a flow

$$
\frac{\partial}{\partial t} \nabla^{2} \Psi+\boldsymbol{u} \cdot \nabla\left[\nabla^{2} \Psi+\beta y+\frac{f_{0} b}{H}\right]=R \nabla^{2}\left(\Psi^{*}-\Psi\right)
$$

needs an additional constraint to determine the boundary values of the streamfunction $\Psi$ on the channel walls. The vorticity concept has eliminated the pressure field and its reconstruction in a multiconnected domain requires in addition to (158) the validity of the momentum balance, integrated over the whole domain,

$$
\frac{\partial U}{\partial t}=R\left(U^{*}-U\right)+\frac{f_{0}}{H}<b \frac{\partial \Psi}{\partial x}>
$$


Here, $U$ is the zonally and meridionally averaged zonal velocity and $R \nabla^{2} \Psi^{*}=$ $-R \partial U^{*} / \partial y$ is the vorticity and $R U^{*}$ the zonal momentum imparted into the system, e.g. by thermal forcing or, in an oceanic application, by wind stress. Furthermore, $R$ is a coefficient of linear bottom friction. The last term in the latter equation is the force exerted by the pressure on the bottom relief, called bottom form stress (the cornered brackets denoted the average over the channel domain). The momentum input $R U^{*}$ is thus balanced by bottom friction and bottom form stress.

The depth of the fluid is $H-b$ and the topography height $b$ is taken sinusoidal, $b=b_{0} \cos K x \sin K y$ with $K=2 \pi n / L$ where $L$ is the length and $L / 2$ the width of the channel. A heavily truncated expansion

$$
\Psi=-U y+\frac{1}{K}[A \cos K x+B \sin K x] \sin K y
$$

represents the flow in terms of the zonal mean $U$ and a wave component with sine and cosine amplitudes $A$ and $B$. It yields the low-order model

$$
\begin{aligned}
\dot{U} & =R\left(U^{*}-U\right)+\frac{1}{4} \delta B \\
\dot{A} & =-K B\left(U-c_{R}\right)-R A \\
\dot{B} & =K A\left(U-c_{R}\right)-\frac{1}{2} \delta U-R B .
\end{aligned}
$$

where $c_{R}=\beta / 2 K^{2}$ is the barotropic Rossby wave speed and $\delta=f_{0} b_{0} / H$. The steady states are readily determined: the wave equations yield for the form stress (the wave component which is out of phase with respect to the topography)

$$
\frac{1}{4} \delta B[U]=-\frac{1}{2} \frac{R \delta^{2} U}{R^{2}+K^{2}\left(U-c_{R}\right)^{2}}
$$

and equating this with $R\left(U^{*}-U\right)$, three equilibria are found if $U^{*}$ is well above $c_{R}$. The three possible steady states can be classified according to the size of the mean flow $U$ compared to the wave amplitudes: the high zonal index regime is frictionally controlled, the flow is intense and the wave amplitude is low; the low zonal index regime is controlled by form stress, the mean flow is weak and the wave is intense. The intermediate state is transitional, it is actually unstable to perturbations. This 'form stress instability' works obviously when the slope of the resonance curve is below the one associated with friction, i.e. $\partial\left(R U-\frac{1}{4} \delta B[U]\right) / \partial U>0$, so that a perturbation must run away from the steady state. Typical parameter values for this model are $R=10^{-6} \mathrm{~s}^{-1}, K=2 \pi / L, L=10000 \mathrm{~km}, b_{0}=500 \mathrm{~m}, H=$ $5000 \mathrm{~m}, U^{*}=60 \mathrm{~ms}^{-1}$. Stochastic versions of the CdV-model have been studied by Egger (1982) and De Swart and Grasman (1987). 
In realistic atmospheric applications of the $\mathrm{CdV}$ model the parameter window (topographic height, forcing and friction parameters) for multiple solutions is quite narrow, due to the dispersiveness of the barotropic Rossby wave it may even not exist at all for more complex topographies where the resonance gets blurred because $c_{R}$ is a function of wave length. For realistic values of oceanic parameters multiple states do not exist because here $U^{*} \ll c_{R}$. Extending the model, however, to baroclinic conditions (a two layer quasigeostrophic model described by (80)), interesting behavior is found which can be applied to the dynamical regime of the Antarctic Circumpolar Current (Olbers and Völker 1996). The Circumpolar Current is due to its zonal unboundedness the only oceanic counterpart (with dynamic similarity) of the zonal atmospheric circulation. The resonance occurs when the barotropic current $U$ is of order of the baroclinic Rossby wave speed $\beta \lambda^{2}$. The model allows for complex topographies since long baroclinic Rossby waves are free of dispersion, the location of the resonance is thus independent of the wavenumber $K$.

In its simplest form the model is derived by expanding the barotropic and baroclinic streamfunctions $\Psi=\Psi_{1}+\Psi_{2}$ and $\Theta=\Psi_{1}-\Psi_{2}$ (assuming equal layer depths for simplicity) again into a small number of modes:

$$
\begin{aligned}
\Psi & =-U y+E \sin 2 y+2[A \cos x+B \sin x] \sin y \\
\Theta & =-u y+G+F \sin 2 y+2[C \cos x+D \sin x] \sin y .
\end{aligned}
$$

All variables are scaled using a time scale $1 /\left|f_{0}\right|$ and a length scale $Y / \pi$ where $Y$ is the channel width. From constraints on the zonal momentum balance similar to (151) and the condition of no mass exchange between the layers one easily arrives at the conditions $E=U / 2, F=u / 2$ and $G=u \pi / 2$ that can be used to eliminate these variables. Inserting the expansion into the potential vorticity balances (81) and projection then yields prognostic equations for $U, u, A, B, C$, and $D$. These are, however, strongly simplified by neglecting the relative vorticity term $\nabla^{2} \Psi_{i}$ and the surface elevation $\zeta$ in the potential vorticity. This approximation is equivalent to reducing the dynamics to the slow baroclinic mode alone, assuming infinitely fast relaxation of the barotropic mode (the fast mode is 'slaved' by the slow mode). Due to this approximation the barotropic low-order equations become diagnostic relations

$$
\begin{aligned}
& 0=-\epsilon(U-u)+b(A-C)+\tau \\
& 0=-\epsilon(A-C)-\beta B-\frac{3}{4} b(U-u) \\
& 0=-\epsilon(B-D)+\beta A,
\end{aligned}
$$


while the baroclinic ones still contain a time derivative,

$$
\begin{aligned}
\frac{3}{2} \dot{u} & =-4 \sigma \mu u+2 \sigma \tau-2(A D-B C) \\
\dot{C} & =-4 \sigma \mu C-\sigma \beta(B+D)+\frac{3}{2}(U D-u B) \\
\dot{D} & =-4 \sigma \mu D+\sigma \beta(A+C)-\frac{3}{2}(U C-u A) .
\end{aligned}
$$

Here, $\epsilon=R /\left|f_{0}\right|$ is the scaled coefficient of a linear bottom friction, $\mu$ is the scaled coefficient of a linear interfacial friction that is meant to mimic the momentum exchange between the layers caused by small-scale eddies (see section 3.4), $\beta=\beta^{*} Y /\left(\pi\left|f_{0}\right|\right)$ is the scaled form of the dimensioned gradient $\beta^{*}$ of the Coriolis parameter, and $\sigma=Y^{2} /(\pi \lambda)^{2}$ is the scaled squared inverse of the baroclinic Rossby radius $\lambda$. The system is forced by a zonal wind stress with scaled amplitude $\tau=\tau_{0} /\left(H Y f_{0}^{2}\right)$ where $\tau_{0}$ is the dimensioned stress amplitude, the meridional dependence is given by $\tau^{x}=\tau \sin ^{2} y$. The scaled height is defined as $b=-(\pi / 2) b_{0} / H$ with the same topography as before in the barotropic CdV-model. Typical parameter values are $\epsilon=10^{-3}, r=2 \epsilon, b_{0}=600 \mathrm{~m}, H=5000 \mathrm{~m}, Y=1500 \mathrm{~km}, \lambda=$ $31 \mathrm{~km}, \tau_{0}=10^{-4} \mathrm{~m}^{2} \mathrm{~s}^{-2}$. The system produces aperiodic oscillations, it contains parameter windows with chaotic behavior (there is Shil'nikov attractor in the range $\left.\mu=2 \cdots 3 \times 10^{-3}, b_{0}=600 \cdots 700 \mathrm{~m}\right)$.

Further reading: Ghil and Childress (1987), James (1994), Völker (1999)

\subsection{Low order models of the thermohaline circulation}

Stommel in his seminal paper of 1961 was the first to point out that the thermohaline circulation in the ocean might have more than one state in equilibrium with the same forcing by input of heat and freshwater at the surface. The notion has found ample interest in recent years in context with the ocean's role in climate change. Numerous papers have demonstrated Stommel's mechanisms with numerical 2-d and 3-d circulation models (see e.g. Marotzke et al. 1998, Rahmstorf et al. 1996).

Stommel's simple model is a two-box representation of the thermal and haline state in the midlatitude and polar regions in the North Atlantic. In terms of the differences $\Delta \theta$ and $\Delta S$ of the temperatures and salinities of the two boxes (well mixed and of equal volume) the evolution equations are

$$
\begin{aligned}
\frac{d \Delta \theta}{d t} & =\gamma\left(\Delta \theta^{*}-\Delta \theta\right)-2|q| \Delta \theta \\
\frac{d \Delta S}{d t} & =2 F-2|q| \Delta S .
\end{aligned}
$$


It is assumed that the flow rate between the boxes is proportional to their density difference,

$$
q=k \Delta \rho=k(-\alpha \Delta \theta+\beta \Delta S),
$$

which assumes hydraulic dynamics where the flow is proportional to pressure (and thus density) differences. Surface heating is parameterized by restoring to an atmospheric temperature $\Delta \theta^{*}$ whereas salt changes are due to a prescribed flux $F$ of freshwater (it is actually virtual flux of salinity related to freshwater flux $E$ (in $\mathrm{ms}^{-1}$ ) by $F=S_{0} E / H$ where $S_{0}$ is constant reference value of salinity and $H$ is the ocean depth; the factor 2 arises because the amount $F$ is taken out of the southern box and imparted into the northern box). These 'mixed' boundary conditions reflect the fact that heat exchange with the atmosphere depends on the sea surface temperature but the freshwater flux $E$ from the atmosphere to the ocean does not depend on the water's salinity. Besides the forcing $F$ there are two more parameters in the model: $\gamma$ is the inverse of a thermal relaxation time, and the hydraulic coefficient $k$ measures the strength of the overturning circulation ( $\alpha$ and $\beta$ are the thermal and haline expansion coefficients of sea water). The ratio $\gamma / q$ determines the role of temperature in this model. If $\gamma \gg q$ the temperature $\Delta \theta$ adjusts very quickly to the atmospheric value $\Delta \theta^{*}$ and the salinity balance alone determines the dynamical system. It has, however, still interesting properties, in particular multiple equilibria. With $\gamma \sim q$ we get a truly coupled thermohaline circulation. Typical parameter values are $k=2 \times 10^{-8} s^{-1}, \alpha=1.8 \times 10^{-4} K^{-1}, \beta=0.8 \times 10^{-3}, \Delta \theta^{*}=10 \mathrm{~K}, F=10^{-14} s^{-1}$. Evidently, $\gamma$ or $k$ may be eliminated by appropriate time scaling.

Steady states are easily found: for $F>0$ (net precipitation in the polar box) there are three equilibria, two of which are stable: a fast flowing circulation (poleward in the surface layer with sinking in the polar box) driven mainly by temperature contrast, and a slow circulation flowing reversely (sinking in the tropics) which is driven by salinity contrast. There is a threshold for $F$ where a saddle-node bifurcation occurs which leaves only the haline mode alive. A detailed description of a phase space perspective of Stommel's model has recently been given by Lohmann and Schneider (1999), noise induced transitions are investigated in Timmermann and Lohmann (1999).

There is no limit cycle associated with the unstable steady state in Stommel's model and the system cannot support self-sustained oscillations. Various routes may be pursued to refine the model, in fact there is great variety of simple thermohaline oscillators which have, in an idealized fashion, a relation to processes in the coupled ocean-atmosphere system. A gallery of thermal and thermohaline oscillators has been collected by Welander (1986).

Presentation of the ocean by more boxes increases the structural complexity (see e.g. Welander 1986, Marotzke 1996, Rahmstorf et al. 1996, Kagan and Maslova 1991) but does not increase the physical content. An obvious weakness lies in the 
representation of the 'hydraulic dynamics' (167) of the circulation relating the meridional flow $q$ to the meridional density gradient $\Delta \rho$, which is contrary to the notion of the geostrophic balance (but it parallels the 2-d closure (133)). A qualitative improvement can be seen in the low-order model of Maas (1994) who considers more complete dynamics in form of the conservation of the 3-d angular momentum

$$
\boldsymbol{L}=\frac{1}{V} \int \boldsymbol{X} \times \boldsymbol{V} d^{3} x
$$

of a rectangular box ocean with volume $V$ and size $L, B, H$ in the $x, y, z$-direction. The coordinate system has its origin in the center ( $x$ eastward, $y$ northward, $z$ upward). The rate of change of angular momentum can be derived from (5) by straightforward integration (we use here the full 3-d balance of momentum but employ the rest of the Boussinesq approximation, i.e. extract the hydrostatically balanced reference field and replace the density in the inertial terms by a constant). A balance by torques due to Coriolis, pressure, buoyancy and frictional forces arises. Under quite 'mild' assumptions and with representation of the density field by plane isopycnal surfaces,

$$
\rho=x \bar{\rho}_{x}(t)+y \bar{\rho}_{y}(t)+z \bar{\rho}_{z}(t)+\left(x^{2}-L^{2} / 3\right) \bar{\rho}_{x x}(t) \cdots
$$

(the last term and all of higher order are ignored), Maas derives a set of six autonomous coupled equations for the vectors $L_{i}$ and $R_{i}=\nabla \bar{\rho}=\int \boldsymbol{x}_{i} \rho d^{3} x / \int x_{i}^{2} d^{3} x$. These are

$$
\begin{aligned}
\operatorname{Pr}^{-1} \dot{\boldsymbol{L}}+\mathrm{Ek}^{-1} \boldsymbol{k} \times \boldsymbol{L} & =-R_{2} \boldsymbol{i}+R_{1} \boldsymbol{j}-\left(L_{1}, L_{2}, r L_{3}\right)+\mathrm{T} \boldsymbol{T} \\
\dot{\boldsymbol{R}}+\boldsymbol{R} \times \boldsymbol{L} & =-\left(R_{1}, R_{2}, \mu R_{3}\right)+\operatorname{Ra} \boldsymbol{Q} .
\end{aligned}
$$

Scaling has been applied: $\operatorname{Pr}=A_{h} /\left(12 K_{h}\right)$ is a Prandtl number, Ek $=2 A_{h} /\left(f L^{2}\right)$ is an Ekman number, $\mu=K_{v} L^{2} /\left(K_{h} H^{2}\right)$ and $r=A_{v} L^{2} /\left(A_{h} H^{2}\right)$ are diffusive and frictional coefficients, $\mathrm{Ra}=g \delta \varrho_{e} H L^{2} /\left(2 A_{h} K_{h}\right)$ is a Rayleigh number (it measures the buoyancy input by an externally imposed density difference $\delta \varrho_{e}$ ), and $\mathrm{T}=$ $\tau_{0} L^{3} /\left(2 H A_{h} K_{h}\right)$ measures the torque exerted by the wind stress $\boldsymbol{\tau}_{0}$. Time is scaled by $L^{2} /\left(12 K_{h}\right)$. The scaled forcing moments are 


$$
\begin{aligned}
\boldsymbol{T} & =\int\left(-\tau_{2} / 2, \tau_{1} / 2,-1\right) d x d y \\
\boldsymbol{Q} & =\int(x, y, 1) B d x d y
\end{aligned}
$$

where $\left(\tau_{1}, \tau_{2}\right)$ is the wind stress vector (scaled by $\tau_{0}$ ) and $B$ the buoyancy flux (scaled by $\delta \varrho_{e} H K_{h} / L^{2}$ ) entering a the surface. Typical values of the dimensionless numbers are $\operatorname{Pr}=2 \times 10^{4}, \mathrm{Ek}=0.02, r=1, \mu=1, \mathrm{Ra}=10^{6}, \mathrm{~T}=500$, the time scale $L^{2} / 12 K_{h}$ is of order of 500 years.

Evidently, the system is easily expanded to nine equations in case that temperature and salinity are used to replace the combined density balance (171) (see Schrier and Maas, 1998). We also point out that in the balance of the zonal component $L_{1}$ of angular momentum we indeed find the terms of the simplified Stommel dynamics (167): neglecting the rate of change and the Coriolis and wind moments we have $L_{1} \approx-R_{2}$ which appears here as the frictional balance between the meridional overturning and the north-south density gradient.

Maas finds a rich suite of regimes in his model. For the case $f=0, \boldsymbol{T}=$ $0, Q_{1}=0$ the Lorenz'63 attractor is found, for the case $\boldsymbol{T}=0, Q_{1}=0, \operatorname{Pr} \rightarrow \infty$ the Lorenz' 84 attractor is found. Typical parameter values for the ocean avoid the chaos which these equation may exhibit. However, multiple equilibria are possible and self-sustained oscillations (with interesting phase portraits) with time scales of order 500 years are obtained.

Further reading: Colin de Verdiere (1993)

\subsection{The delayed ENSO oscillator}

Simple conceptual models of the oscillatory behavior of ENSO (cf. section 4.4) were suggested by Suarez and Schopf (1988) and Battisti and Hirst (1989). They are represented by a one-dimensional state variable $T$ which could be any of the variables of the coupled ocean-atmosphere ENSO system, for instance the thermocline depth anomaly or the anomalies of the sea surface temperature or the wind stress amplitude. The model combines the physics of wave propagation and the unstable coupled mode, explained in the sections 3.1 and 4.4, into one equation. An example is the delayed oscillator

$$
\dot{T}=c T-b T(t-\tau)-e T^{3} .
$$

The first term on the rhs represents the positive feedback (with $c>0$ ), the second term the delayed effect of the Kelvin and Rossby wave propagation across the equatorial basin. The Rossby waves are excited in the interaction region, later reflected at the western boundary and then - after the time delay $\tau$ - return 
their signal to the east via a Kelvin wave. The cubic term limits the growing unstable mode. Two of the four parameters $(c$ and $e$ ) can be eliminated by scaling time by $c$ and $T$ by $\sqrt{c / e}$. Depending on the values of the remaining parameters, $b / c$ and $c \tau$, steady or oscillatory solutions are possible (a detailed discussion of (173) and other low-order models of ENSO is given by McCreary and Anderson (1991), take e.g. $c=1, e=1, b=0.5, \tau=1$ for a steady case, or $c=1, e=$ $1, b=1.5, \tau=3$ for oscillating case). Note that these models can be extended to seasonally varying parameters. The solution is very sensitive to the parameters so that in case of stochastic forcing an interesting switching of regimes occurs which might be relevant to the ENSO phenomenon.

Acknowledgements I appreciate discussions with Hartmut Borth, Andreas Hense, Mojib Latif, Christoph Völker and, in particular, with Jürgen Willebrand. Josef Egger and an anonymous reviewer helped to improve the manuscript. I am grateful to Klaus Hasselmann, my teacher in physics over three decades. Contribution No. 1697 from the Alfred-Wegener-Institute for Polar and Marine Research.

\section{References}

Battisti, D.S., and A.C. Hirst, 1989: Interannual variability in the tropical atmosphere/ocean system: influence of basic state, ocean geometry and nonlinearity, $J$. Atmos. Sciences, 46, 1687-1712.

Benzi, R., Saltzman, B., and A. Wijn-Nielsen, 1986: Anomalous atmospheric flows and blocking, Academic Press, Orlando.

Bolin, B., 1955: Numerical forcasting with the barotropic model, Tellus, 7, 27-49.

Broecker, W.S., 1991: The great ocean conveyor, Oceanography, 4, 79-89.

Chandrasekhar, S., 1961: Hydrodynamic and hydromagnetic stability, Dover Publications, Inc., New York.

Charney, J.G., 1955: The use of the primitive equations of motion in numerical prediction, Tellus, 7, 22-26.

Charney, J.G. and J.G. DeVore, 1979: Multiple flow equilibra in the atmosphere and blocking, J. Atmos. Sciences, 36, $1205-1216$.

Colin de Verdiere, A., 1993: On the thermohaline circulation, in Modelling Ocean Climate Interactions, edited by J. Willebrand, and D. Anderson, pp. 151-183, Berlin, Springer Verlag.

De Swart, H.E., and J. Grasman, 1987: Effect of stochastic perturbations on a low-order spectral model of the atmospheric circulation, Tellus, 39A, 10-24.

Eckert, C., and M. Latif, 1997: Predictability of a stochastically forced hybrid coupled model of El Niño, J. Climate, 10, 1488-1504.

Egger, J., 1978: Dynamics of blocking highs, J. Atmos. Sciences, 35, 1788-1801.

Egger, J., 1982: Stochastically driven large-scale circulations with multiple equilibria, J. Atmos. Sciences, 38, 2606-2618.

Egger, J., 1999: POPs and MOPs, Climate Dynamics, 15, 561-568.

Frankignoul, C., 1995: Climate spectra and stochastic climate models, in Analysis of climate variability. Application of statistical techniques, edited by $\mathrm{H}$. von Storch, and A. Navarra, pp. 29-51, Berlin, Springer Verlag. 
Frankignoul, C., 1999: Sea surface temperature variability in the North Atlantic: monthly to decadal time scales, in Beyond El Niño, edited by A. Navarra, pp. 25-48, Berlin, Springer Verlag.

Frankignoul, C. and K. Hasselmann, 1977: Stochastic climate models, Part II. Application to sea surface temperature anomalies and thermocline variability, Tellus, 29, 289-305.

Frankignoul, C., Müller, P., and E. Zorita, 1997: A simple model of the decadal response of the ocean to stochastic wind forcing, J. Phys. Oceanogr., 27, 1533-1546.

Gent, P.R., and J.C. McWilliams, 1983a: Consistent balanced models in bounded and periodic domains, Dyn. Atmos. Oceans, 7, 63-97.

Gent, P.R., and J.C. McWilliams, 1983b: Regimes of validity for balanced models, Dyn. Atmos. Oceans, 7, 167-183.

Ghil, M., 1981: Energy-balance models: an introduction, in Climate variations and variability: facts and theory, edited by A. Berger, 461-480, D. Reidel Publishing Conpany.

Ghil, M., and S. Childress, 1987: Topics in geophysical fluid dynamics: atmospheric dynamics, dynamo theory, and climate dynamics, Springer Verlag, Berlin.

Gill, A.E., 1982: Atmosphere-Ocean Dynamics, Academic Press, New York.

Goldsbrough, G.R., 1933: Ocean currents produced by evaporation and precipitation, Proc. Roy. Soc. London, A141, 512-517.

Goldsbrough, G.R., 1934: On ocean currents produced by winds, Proc. Roy. Soc. London, A148, 47-58.

Hasselmann, K., 1970: Wave-driven inertial oscillations, Geophys. Fluid Dyn., 1, 463-502.

Hasselmann, K., 1976: Stochastic climate models, Part I: Theory, Tellus, 28, 473-485.

Held, I.M., and A.Y. Hou, 1980: Nonlinear axially symmetric circulations in a nearly inviscid atmosphere, J. Atmos. Sci., 37, 515-533.

James, I.N., 1994: Introduction to circulating atmospheres, Cambridge University Press, Cambridge.

Kagan, B.A., and N.B. Maslova, 1991: Non-uniqueness of the thermohaline circulation in a three-layer ventilated ocean, Fluid Dyn. Res., 8, 287-295.

Källen, E., Crafoord, C., and M. Ghil, 1980: Free Oscillations in a climate model with ice-sheet dynamics, J. Atmos. Sciences, 36, 2292-2303.

Kraus, E.B., 1977: Modelling and prediction of the upper layers of the ocean, Pergamon Press, Oxford.

Lemke, P., 1977: Stochastic climate models, Part 3: Application to zonally-averaged energy balance models, Tellus, 29, 385-392.

Lemke, P., 1986: Stochastic description of atmosphere-sea ice-ocean interaction, in The Geophysics of Sea Ice, edited by N. Untersteiner, pp. 785-823, Plenum.

Lemke, P., E.W. Trinkl and K. Hasselmann, 1980: Stochastic dynamic analysis of polar sea ice variability, J. Phys. Oceanogr., 10, 2100-2120.

Lindzen, R. S., 1990: Dynamics of Atmospheric Physics, Cambridge University Press.

Lohmann, G., and J. Schneider, 1999: Dynamics and predictability of Stommel's box model. A phase-space perspective with implications for decadal climate variability, Tellus, 51A, 326-336.

Lorenz, E.N., 1960: Maximum simplification of the dynamical equations, Tellus, 12, 243254.

Lorenz, E.N., 1963: Deterministic Nonperiodic Flow, J. Atmos. Sciences, 20, 130-141.

Lorenz, E.N., 1967: The nature and theory of the general ciculation of the atmosphere, Tech. rep., p. 161, World Meteorological Organisation. 
Lorenz, E.N., 1984: Irregularity: a fundamental property of the atmosphere, Tellus, 36A, 98-110.

Lovelock, J. E., 1989: Geophysiology, the Science of Gaia, Rev. Geophys., 27(2), 215-222.

Maas, L.R.M., 1994: A simple model for the three-dimensional, thermally and winddriven ocean circulation, Tellus, 46A, 671-680.

Marotzke, J., P. Welander and J. Willebrand, 1988: Instability and multiple steady states in a meridional-plane model of the thermohaline circulation, Tellus, 40A, 162-172.

McCreary, J.P., and D.L.T. Anderson, 1991: An overview of coupled ocean-atmosphere models of El Niño and the Southern Oscillation, J. Geophys. Res., 96(Suppl.), 31253150 .

Mikolajewicz, U., and E. Maier-Reimer, 1990: Internal secular variability in an OGCM, Climate Dyn., 4, 145-156.

Müller, P., and J. Willebrand, 1989: Equations of oceanic motions, in Landolt-Börnstein Numerical Data and Functional Relationships in Science and Technology - New Series, Group V, Volume 3b, edited by J. Sündermann, pp. 1-14, Berlin, Springer Verlag.

Munk, W.H., 1950: On the wind-driven ocean circulation, J. Meteorol., 7(2), 79-93.

Navarra, A., 1999: Beyond El Niño, Springer Verlag, Berlin.

Needler, G.T., 1967: A model for thermohaline circulation in an ocean of finite depth, $J$. Mar. Res., 25, 329-342.

Neelin, J.D., Latif, M., and F.-F. Jin, 1994: Dynamics of coupled ocean-atmosphere models: the tropical problem, Ann. Rev. Fluid Mech., 26, 617-659.

North, G. R., 1981: Energy Balance Models, Rev. Geophys., 19, 91-121.

Olbers, D., and Chr. Völker, 1996: Steady states and variability in oceanic zonal flows, in Decadal climate variability dynamics and predicition, edited by D. A. and J. Willebrand, pp. 407-443, Berlin, Springer-Verlag.

Olbers, D., and J.-O. Wolff, 2000: A low-order model of baroclinic channel flow over topography - with applications to the ACC, in preparation.

Olbers, D., Lemke, P., and D. Wolf-Gladrow, 1997: Physik des Klimasystems, AlfredWegener-Institute, Bremerhaven.

Olbers, D., Willebrand, J., and P. Lemke, 1999: Fundamentals and concepts of ocean dynamics and thermodynamics, Alfred-Wegener-Institute, Bremerhaven.

Olbers, D.J., 1986: Internal Waves, in Landolt-Börnstein - Numerical Data and Functional Relationships in Science and Technology - New Series, Group V, Volume 3a, edited by Sündermann, J., pp. 37-82, Springer, Berlin.

Palmer, T.N., 1998: Nonlinear dynamics and climate change: Rossby's legacy, Bull. Am. Met Soc., 79(7), 1411-1423.

Pavan, V., and I.M. Held, 1996: The diffusive approximation of eddy fluxes in baroclinically unstable jets, J. Atmos. Sciences, 53, 1262-1272.

Pedlosky, J., 1987a: Geophysical Fluid Dynamics, Springer-Verlag, Berlin.

Pedlosky, J., 1987b: Thermocline theories, in General Circulation of the Ocean, edited by H.D.I. Abarbanel and W.R. Young, pp. 55-101, Springer Verlag, Berlin.

Peixoto, J.P., and A.H. Oort, 1992: Physics of climate, American Institute of Physics, New York.

Philander, S.G., 1990: El Niño, La Niña, and the Southern Oscillation, Academic Press, New York.

Rahmstorf, S., 1995: Bifurcations of the Atlantic thermohaline circulation in response to changes in the hydrological cycle, Nature, 378, 145-149. 
Rahmstorf, S., Marotzke, M., and J. Willebrand, 1996: Stability of the Thermohaline Circulation, in The Warmwatersphere of the North Atlantic Ocean, edited by W. Krauss, pp. 129-157, Berlin, Gebr. Borntraeger.

Ramanathan, V., and J.A. Coakley, 1978: Climate modeling through radiative-convective models, Rev. Geophys. Space Phys., 16, 465-489.

Salby, M.L., 1996: Fundamentals of Atmospheric Physics, Academic Press, New York.

Salmon, R., 1998: Lectures on Geophysical Fluid Dynamics, Oxford University Press, Oxford.

Schneider, E., and R.S. Lindzen, 1977: Axial symmetric steady state models of the basic state of instability and climate studies. Part I: Linearized calculations, J. Atmos. Sci., 34, 253-279.

Schrier, G. van der, and L.R.M. Maas, 1998: Chaos in a simple model of the threedimensional, salt-dominated ocean circulation, Climate Dyn., 14, 489-502.

Stocker, T.F., D.G. Wright, and L.A. Mysak, 1992: A Zonally Averaged, Coupled OceanAtmosphere Model for Paleoclimate Studies, J. Climate, 5, 773-797.

Stommel, H., 1948: The westward intensification of wind-driven currents, Trans. Am. Geophys. Union, 29, 202-206.

Stommel, H., 1961: Thermohaline convection with two stable regimes of flow, Tellus, 13, $224-230$.

Stommel, H., 1984: The delicate interplay between wind-stress and buoyancy input in ocean circulation: the Goldsbrough variations, Tellus, 36A, 111-119.

Suarez, M.J., and P.S. Schopf, 1988: A delayed action oscillator for ENSO, J. Atmos. Sciences, 45, 3283-3287.

Timmermann, A., and G. Lohmann, 1999: Noise-induced transitions in a simplified model of the thermohaline circulation, J. Phys. Oceanogr., in press.

Trenberth, K.E. (Ed.), 1992: Climate system modeling, Cambridge University Press, Cambridge.

Tung, K.K., and A.J. Rosenthal, 1985: Theories of multiple equilibria - a critical reexamination. Part i: barotropic models, J. Atmos. Sciences, 42, 2804-2819.

Veronis, G., 1963: An analysis of wind-driven ocean circulation with a limited number of Fourier components, J. Atmos. Sciences, 20, 577-593.

Völker, C, 1999: Momentum balance in zonal flows and resonance of baroclinic Rossby waves, J. Phys. Oceanogr., 29, 1666-1681.

Washington, W.M., and C.L. Parkinson, 1986: An introduction to three-dimensional climate modeling, Oxford University Press, Oxford.

Watson, A. J., and J. E. Lovelock, 1983: Biological homeostasis of the global environment: the parable of Daisyworld, Tellus, 35B, 284-289.

Welander, P., 1971: Some Exact Solutions to the Equations Describing the Ideal Fluid Thermocline, J. Mar. Res., 29, 60-68.

Welander, P., 1986: Thermohaline effects in the ocean circulation and related simple models, in Large-Scale Transport Processes in Oceans and Atmosphere, edited by J. Willebrand, and D. Anderson, pp. 163-200, D. Reidel.

Wolff, J.-O., Maier-Reimer, E. and Olbers, D.J., 1991: Wind-Driven Flow over Topography in a Zonal $\beta$-Plane Channel: A Quasi-geostrophic model of the Antarctic Circumpolar Current, J. Phys. Oceanogr., 21(2), 236-264. 
Wright, D.G. and T.F. Stocker, 1991: A zonally averaged ocean for the thermohaline circulation. Part I: Model development and flow dynamics, J. Phys. Oceanogr., 21, 1713-1724.

Zebiak, S.E., and M.A. Cane, 1987: A model El Niño-Southern Oscillation, Mon. Wea. Rev., 115, 2262-2278. 


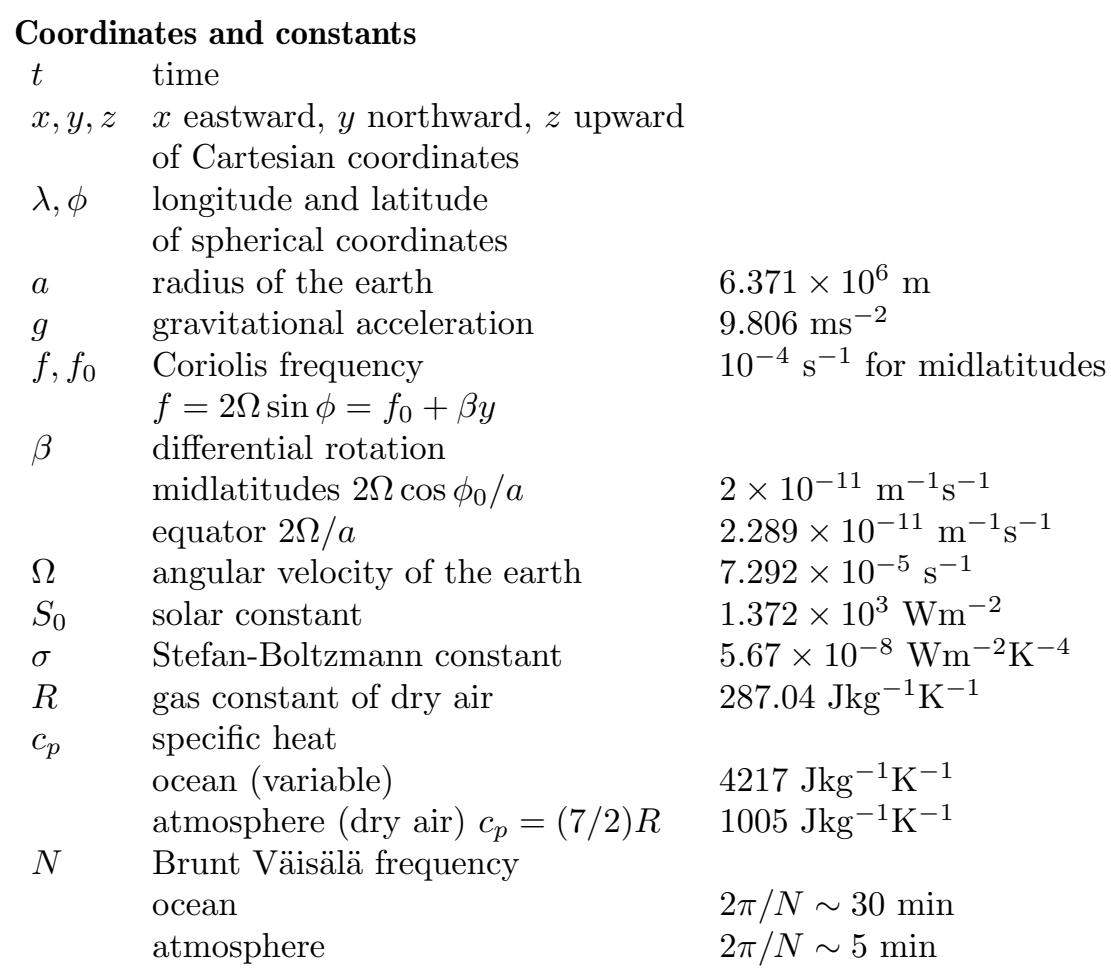

The forcing functions of the wave equations

$$
\begin{aligned}
\left(\mathcal{F}^{u}, \mathcal{F}^{v}\right) & =\mathbf{\Phi} / \varrho_{0}-\boldsymbol{u} \cdot \nabla \boldsymbol{u}-w \frac{\partial \boldsymbol{u}}{\partial z} \\
\mathcal{G}_{b} & =-\left(g / \varrho_{0}\right)\left[-\alpha_{*} \Gamma_{\theta}+\beta_{*} \Gamma_{S}\right]-\boldsymbol{u} \cdot \nabla b-w \frac{\partial b}{\partial z} \\
\mathcal{P} & =-\left(\zeta \frac{\partial p}{\partial z}+\frac{1}{2} N^{2} \zeta^{2}+\cdots\right)_{z=0}+p_{a t m} \\
\mathcal{Z} & =\left(\zeta \frac{\partial w}{\partial z}+\cdots\right)_{z=0} \\
\mathcal{W} & =\left(\boldsymbol{u} \cdot \nabla \eta-\eta \frac{\partial w}{\partial z}-\cdots\right)_{z=-H} \\
\mathcal{Q} & =\frac{\partial \mathcal{P}}{\partial t}+g^{2} \mathcal{Z}-\int_{z}^{0} \mathcal{G}_{b} d z \\
\mathcal{C} & =\frac{\partial \mathcal{F}^{v}}{\partial x}-\frac{\partial \mathcal{F}^{u}}{\partial y}-\frac{1}{f_{0}} \mathcal{M}^{-1} \mathcal{Q} \\
\mathcal{D} & =\frac{\partial \mathcal{F}^{u}}{\partial x}+\frac{\partial \mathcal{F}^{v}}{\partial y}
\end{aligned}
$$


where $h=H-\eta$, i.e. $\eta$ is the elevation of the bottom above the mean depth $H$.

Dirk Olbers

Alfred-Wegener-Institute for Polar and Marine Research

25757 Bremerhaven, Germany

E-mail address: dolbers@awi-bremerhaven.de 